Proceedings of the Edinburgh Mathematical Society (2007) 50, 429-457 (C)

DOI:10.1017/S0013091505001495 Printed in the United Kingdom

\title{
A CLASS OF STRONGLY SINGULAR RADON TRANSFORMS ON THE HEISENBERG GROUP
}

\author{
NEIL LYALL* \\ Scuola Normale Superiore, Piazza dei Cavalieri 7, 56126 Pisa, Italy
}

(Received 2 November 2005)

\begin{abstract}
We primarily consider here the $L^{2}$ mapping properties of a class of strongly singular Radon transforms on the Heisenberg group $\mathbb{H}^{n}$; these are convolution operators on $\mathbb{H}^{n}$ with kernels of the form $M(z, t)=K(z) \delta_{0}(t)$, where $K$ is a strongly singular kernel on $\mathbb{C}^{n}$. Our results are obtained by using the group Fourier transform and uniform asymptotic forms for Laguerre functions due to Erdélyi.

We also discuss the behaviour of related twisted strongly singular operators on $L^{2}\left(\mathbb{C}^{n}\right)$ and obtain results in this context independently of group Fourier transform methods. Key to this argument is a generalization of the results for classical strongly singular integrals on $L^{2}\left(\mathbb{R}^{d}\right)$.
\end{abstract}

Keywords: strongly singular integral; Radon transform; Heisenberg group

2000 Mathematics subject classification: Primary 44A12; 42B20; 43A80

\section{Introduction}

In this article our main consideration will be the $L^{2}$ mapping properties of a class of strongly singular Radon transforms on the Heisenberg group $\mathbb{H}^{n}$. More precisely, we consider convolution operators on $\mathbb{H}^{n}$ with kernels of the form $M(z, t)=K(z) \delta_{0}(t)$, where $K$ is a kernel on $\mathbb{C}^{n}$ that is too singular at the origin to be of Calderón-Zygmund type and that has this strong singularity compensated for by the introduction of a suitably large oscillation. Our main result is stated in $\S 1.2$ and is obtained by using group Fourier transform methods and uniform asymptotic forms for Laguerre functions due to Erdélyi [3].

We also discuss the behaviour of related twisted strongly singular integral operators on $L^{2}\left(\mathbb{C}^{n}\right)$. These results are stated in $\S 1.3$ and are obtained independently of group Fourier transform techniques. Key to these arguments is a generalization of existing results for classical strongly singular integrals on $L^{2}\left(\mathbb{R}^{d}\right)$. We choose to state these results first.

\subsection{Strongly singular integrals on $\mathbb{R}^{d}$}

Strongly singular integrals on $\mathbb{R}^{d}$ are operators $T$, initially defined as mappings from test functions in $\mathcal{S}\left(\mathbb{R}^{d}\right)$ to distributions in $\mathcal{S}^{\prime}\left(\mathbb{R}^{d}\right)$, to which are associated kernels

* Present address: Department of Mathematics, University of Georgia, Athens, GA 30602, USA (lyall@math.uga.edu). 
$K_{\alpha, \beta}(x, y)$, defined when $x \neq y$, that take the form

$$
K_{\alpha, \beta}(x, y)=a(x, y) \mathrm{e}^{\mathrm{i} \varphi(x, y)} .
$$

We assume that the amplitude and phase satisfy the differential inequalities

$$
\begin{gathered}
\left|\partial_{x}^{\mu} \partial_{y}^{\nu} a(x, y)\right| \leqslant C_{\mu, \nu}|x-y|^{-d-\alpha-|\mu|-|\nu|}, \\
\left|\partial_{x}^{\mu} \partial_{y}^{\nu} \varphi(x, y)\right| \leqslant C_{\mu, \nu}|x-y|^{-\beta-|\mu|-|\nu|}
\end{gathered}
$$

that $\varphi$ is real valued and, furthermore, that

$$
\left|\nabla_{x} \varphi(x, y)\right|,\left|\nabla_{y} \varphi(x, y)\right| \geqslant C|x-y|^{-\beta-1}
$$

with $\alpha \geqslant 0$ and $\beta>0$. In the case where $\alpha=0$, we must make the further assumption that our amplitude $a$ is compactly supported in a neighbourhood of the diagonal $x=y$; this is of course also the only region of interest when $\alpha>0$.

It is clear that the estimates (1.2) also hold uniformly for the dilated functions

$$
a_{\lambda}(x, y)=\lambda^{d+\alpha} a(\lambda x, \lambda y) \quad \text { and } \quad \varphi_{\lambda}(x, y)=\lambda^{\beta} \varphi(\lambda x, \lambda y),
$$

and, in addition to the differential inequalities (1.2) above, we make the following nondegeneracy assumption, namely that

$$
\left|\operatorname{det}\left(\frac{\partial^{2} \varphi_{\lambda}(x, y)}{\partial x_{i} \partial y_{j}}\right)\right| \geqslant C>0
$$

uniformly in $\lambda$. Such kernels we will call (non-degenerate) strongly singular integral kernels.

Our strongly singular integral operators $T$ are related to these kernels $K_{\alpha, \beta}$ as follows: for $f \in \mathcal{S}$ with compact support, we identify the distribution $T f$ with the function

$$
T f(x)=\int K_{\alpha, \beta}(x, y) f(y) \mathrm{d} y,
$$

for $x$ outside the support of $f$. Our result for such operators is the following.

Theorem 1.1. The operator $T$, initially given by (1.4), extends to a bounded operator on $L^{2}\left(\mathbb{R}^{d}\right)$ if and only if $\alpha \leqslant \frac{1}{2} d \beta$.

The proof of this result is presented in $\S 5$.

The model for operators of this type is those with kernels

$$
K_{\alpha, \beta}(x, y)=\tilde{K}_{\alpha, \beta}(x-y),
$$

where $\tilde{K}_{\alpha, \beta}$ is a distribution* on $\mathbb{R}^{d}$ that, away from the origin, agrees with the radial function

$$
\tilde{K}_{\alpha, \beta}(x)=|x|^{-d-\alpha} \mathrm{e}^{\mathrm{i}|x|^{-\beta}} \chi(|x|),
$$

* The distribution-valued function $\alpha \mapsto \tilde{K}_{\alpha, \beta}$, initially defined for $\operatorname{Re} \alpha<0$, continues analytically to all of $\mathbb{C}$. 
with $\beta>0$ and $\chi$ smooth and compactly supported in a small neighbourhood of the origin.

Operators of this type were first studied by Hirschman [7] in the case $d=1$ and then studied for higher dimensions by Wainger [12], Fefferman [4] and Fefferman and Stein [5]. Proving that these operators are indeed of the form considered in Theorem 1.1, and that their kernels are in particular non-degenerate, is an easy exercise (see $§ 5.2$ ).

To establish Theorem 1.1 in this model case, it is efficient to use Fourier transform methods. Since $\tilde{K}_{\alpha, \beta}$ is a radial compactly supported distribution, it is well known that its Fourier transform is a smooth radial function given by

$$
m(\xi)=(2 \pi)^{d / 2} \int_{0}^{\infty} \chi(r) r^{-1-\alpha} \mathrm{e}^{\mathrm{i} r^{-\beta}} J_{(d-2) / 2}(r|\xi|)(r|\xi|)^{(d-2) / 2} \mathrm{~d} r
$$

where $J_{(d-2) / 2}$ is a Bessel function (see [10]). Using Plancherel's theorem and the asymptotics of Bessel functions, it is then straightforward to establish Theorem 1.1 in this case.

\subsection{The Heisenberg group}

The Heisenberg group $\mathbb{H}^{n}$ is $\mathbb{C}^{n} \times \mathbb{R}$ endowed with the group law

$$
[z, t] \cdot[w, s]=\left[z+w, t+s+\frac{1}{2} \operatorname{Im} z \cdot \bar{w}\right],
$$

with identity the origin and inverses given by $[z, t]^{-1}=[-z,-t]$.

The following transformations are automorphisms of the group $\mathbb{H}^{n}$ :

(i) non-isotropic dilations, $[z, t] \mapsto \delta \circ[z, t]=\left[\delta z, \delta^{2} t\right]$, for all $\delta>0$;

(ii) rotations, $[z, t] \mapsto[U z, t]$, with $U$ a unitary transformation of $\mathbb{C}^{n}$.

The usual Lebesgue measure $\mathrm{d} z \mathrm{~d} t$ on $\mathbb{C}^{n} \times \mathbb{R}$ is the Haar measure for $\mathbb{H}^{n}$.

A natural analogue, in this Heisenberg group setting, to the model operators discussed above has been studied by Lyall $[\mathbf{8}]$.

In this article we will consider the class of strongly singular Radon transforms on the Heisenberg group $\mathbb{H}^{n}$ formally given by

$$
R f(z, t)=\int_{\mathbb{H}^{n}} M\left([w, s]^{-1} \cdot[z, t]\right) f(w, s) \mathrm{d} w \mathrm{~d} s,
$$

where $M$ are distribution kernels of the form

$$
M=\tilde{K}_{\alpha, \beta} \otimes \delta_{0}
$$

with $\tilde{K}_{\alpha, \beta}$ the radial strongly singular kernels on $\mathbb{C}^{n}\left(\mathbb{R}^{d}\right.$ with $\left.d=2 n\right)$ given by $(1.5)$.

Our main result is then the following.

Theorem 1.2. $R$ extends to a bounded operator on $L^{2}\left(\mathbb{H}^{n}\right)$ if and only if $\alpha \leqslant\left(n-\frac{1}{6}\right) \beta$.

We obtain this result via group Fourier transform methods. Similar methods were employed in [8]. However, the arguments in this setting are simpler. 


\subsection{Twisted strongly singular integrals on $\mathbb{C}^{n}$}

The operators above are of course intimately connected with the twisted convolution operators

$$
R^{\lambda} f(z)=\int_{\mathbb{C}^{n}} \tilde{K}_{\alpha, \beta}(z-w) \mathrm{e}^{\mathrm{i}(\lambda / 2) \operatorname{Im} z \cdot \bar{w}} f(w) \mathrm{d} w .
$$

In fact, it follows from taking the partial Fourier transform in the $t$ variable and applying Plancherel's theorem that the boundedness of $R$ on $L^{2}\left(\mathbb{H}^{n}\right)$ is formally equivalent to the uniform boundedness of $R^{\lambda}$ on $L^{2}\left(\mathbb{C}^{n}\right)$ for $\lambda \neq 0$. It is therefore interesting to note the following corollary of the proof of Theorem 1.2.

Corollary 1.3. For any fixed real $\lambda$, the operator $R^{\lambda}$ extends to a bounded operator on $L^{2}\left(\mathbb{C}^{n}\right)$ whenever $\alpha \leqslant n \beta$.

However, we will prove a more general version of this result and do so independently of group Fourier transform methods.

We will consider operators $T^{\lambda}$, initially defined as mappings from test functions in $\mathcal{S}\left(\mathbb{C}^{n}\right)$ to distributions in $\mathcal{S}^{\prime}\left(\mathbb{C}^{n}\right)$, to which we associate strongly singular integral kernels $K_{\alpha, \beta}(z, w)$ on $\mathbb{C}^{n}$.

For $f \in \mathcal{S}$ with compact support, we identify the distribution $T^{\lambda} f$ with the function

$$
T^{\lambda} f(z)=\int_{\mathbb{C}^{n}} K_{\alpha, \beta}(z, w) \mathrm{e}^{\mathrm{i}(\lambda / 2) \operatorname{Im} z \cdot \bar{w}} f(w) \mathrm{d} w,
$$

for $z$ outside the support of $f$. Our result for such operators is the following.

Theorem 1.4. For any $\lambda$ real, $\left\|T^{\lambda} f\right\|_{L^{2}\left(\mathbb{C}^{n}\right)} \leqslant A_{\lambda}\|f\|_{L^{2}\left(\mathbb{C}^{n}\right)}$ if and only if $\alpha \leqslant n \beta$.

\section{Remark 1.5.}

(i) Theorem 1.1 is of course essentially a special case of Theorem 1.4 with $\lambda=0$.

(ii) It would be of interest to know the precise behaviour of the constant $A_{\lambda}$ in Theorem 1.4 as $|\lambda| \rightarrow \infty$.

In the next three sections we will concern ourselves with the proof of Theorem 1.2: in $\S 2$ we introduce the group Fourier transform and reduce matters to basic Laguerre transform estimates; as with the model Euclidean case, the asymptotics of special functions, in this case Laguerre polynomials, will be crucial and we include a discussion of these expansions in $\S 3$; finally, in $\S 4$, we present the proof of these key Laguerre transform estimates.

The proof of Theorem 1.4 is presented in $\S 5$.

\section{Reduction of Theorem 1.2 to Laguerre transform estimates}

Let $\varepsilon>0$ and set $M^{\varepsilon}(z, t)=\tilde{K}_{\alpha, \beta}^{\varepsilon}(z) \delta_{0}(t)$, where

$$
\tilde{K}_{\alpha, \beta}^{\varepsilon}(z)=\mathrm{e}^{-\varepsilon|z|^{-\beta}} \tilde{K}_{\alpha, \beta}(z),
$$


and for $f \in L^{2}\left(\mathbb{H}^{n}\right)$ we define

$$
R^{\varepsilon} f(z, t)=f * M^{\varepsilon}(z, t),
$$

where convolution is taken with respect to the group structure on $\mathbb{H}^{n}$. It is then easy to see that if, for fixed $\varepsilon>0$, we integrate the function $\tilde{K}_{\alpha, \beta}^{\varepsilon}$ by parts $N$ times and take the limit as $\varepsilon \rightarrow 0$, then this must agree with the unique analytic continuation of $\tilde{K}_{\alpha, \beta}$ to the half-plane $\operatorname{Re}(\alpha)<N \beta$. It then follows that, for $f \in \mathcal{S}\left(\mathbb{H}^{n}\right)$,

$$
R f(z, t)=\lim _{\varepsilon \rightarrow 0} R^{\varepsilon} f(z, t) .
$$

We will therefore, in the following, content ourselves with studying the operator $R^{\varepsilon}$.

\subsection{Group Fourier transform}

It follows from Plancherel's theorem for the group Fourier transform that

$$
\left\|R^{\varepsilon} f\right\|_{L^{2}\left(\mathbb{H}^{n}\right)} \leqslant A\|f\|_{L^{2}\left(\mathbb{H}^{n}\right)} \Longleftrightarrow\left\|\hat{M}^{\varepsilon}(\lambda)\right\|_{O p} \leqslant A \text { uniformly over } \lambda \neq 0,
$$

where $\hat{M}^{\varepsilon}(\lambda)$ denotes the group Fourier transform of $M^{\varepsilon}$, which for each $\lambda \neq 0$ is an operator on the Hilbert space $L^{2}\left(\mathbb{R}^{n}\right)$. Now, since the $M^{\varepsilon}$ were chosen to be radial on $\mathbb{H}^{n}$, i.e. $M^{\varepsilon}(z, t)=M_{0}^{\varepsilon}(|z|, t)$ for some function $M_{0}^{\varepsilon}$, it is a well-known result of Geller [6] that the operators $\hat{M}^{\varepsilon}(\lambda)$ are in fact, for each $\lambda \neq 0$, diagonal with respect to a (rescaled) Hermite basis for $L^{2}\left(\mathbb{R}^{n}\right)$. More precisely,

$$
\hat{M}^{\varepsilon}(\lambda)=C_{n}\left(\delta_{\boldsymbol{j}, \boldsymbol{k}} \mu(|\boldsymbol{k}|, \lambda)\right)_{\boldsymbol{j}, \boldsymbol{k} \in \mathbb{N}^{n}}
$$

where $C_{n}$ is a constant which depends only on the dimension, and the diagonal entries $\mu(|\boldsymbol{k}|, \lambda)$ can be expressed explicitly in terms of a Laguerre transform. Setting $k=|\boldsymbol{k}|$, we in fact have

$$
\mu(k, \lambda)=c_{k}^{n-1} \int_{0}^{\infty} \mathrm{e}^{-\varepsilon r^{-\beta}} \chi(r) r^{-1-\alpha} \mathrm{e}^{\mathrm{i} r^{-\beta}} \Lambda_{k}^{n-1}\left(\frac{1}{2}|\lambda| r^{2}\right)\left(\frac{1}{2}|\lambda| r^{2}\right)^{(1-n) / 2} \mathrm{~d} r
$$

where

$$
c_{k}^{\delta}=\left(\frac{k !}{(k+\delta) !}\right)^{1 / 2}
$$

and $\Lambda_{k}^{\delta}(x)$ is a Laguerre function of type $\delta$. Recall that Laguerre functions of type $\delta$, $\delta>-1$, form an orthonormal basis for $L^{2}\left(\mathbb{R}^{+}\right)$and are given by

$$
\Lambda_{k}^{\delta}(x)=c_{k}^{\delta} L_{k}^{\delta}(x) \mathrm{e}^{-x / 2} x^{\delta / 2},
$$

where

$$
L_{k}^{\delta}(x)=\sum_{j=0}^{k}\left(\begin{array}{l}
k+\delta \\
k-j
\end{array}\right) \frac{(-x)^{j}}{j !}
$$

are the Laguerre polynomials of type $\delta$.

It therefore follows that the operators $\hat{M}^{\varepsilon}(\lambda)$ are bounded on $L^{2}\left(\mathbb{R}^{n}\right)$ if and only if the diagonal scalars $\mu(k, \lambda)$ are bounded uniformly in $k$, and hence

$$
\left\|R^{\varepsilon} f\right\|_{L^{2}\left(\mathbb{H}^{n}\right)} \leqslant A\|f\|_{L^{2}\left(\mathbb{H}^{n}\right)} \Longleftrightarrow|\mu(k, \lambda)| \leqslant A^{\prime} \text {, uniformly in } k \text { and } \lambda \neq 0 .
$$

For more on the group Fourier transform and Laguerre functions see $[\mathbf{9}, \mathbf{1 1}]$. 


\subsection{Main estimates}

We have seen that matters reduce to the study of the 'Fourier transforms' $\mu(k, \lambda)$. Our main estimate is then the following.

Theorem 2.1.

(i) If $|\lambda| \leqslant k$, then $|\mu(k, \lambda)| \leqslant c_{0}(1+|\lambda| k)^{(\alpha-n \beta) / 2(\beta+1)}$.

(ii) If $|\lambda| \geqslant k$, then, as $k \rightarrow \infty$,

$$
\begin{aligned}
\mu(k, \lambda)=c_{1}(|\lambda| k)^{(\alpha-(n-1 / 6) \beta) / 2(\beta+1)} & \\
& \times \exp \left\{\mathrm{i} c_{2}(|\lambda| k)^{\beta / 2(\beta+1)}\right\}+O\left((|\lambda| k)^{(\alpha-n \beta) / 2(\beta+1)}\right) .
\end{aligned}
$$

The constants $c_{0}, c_{1}$ and $c_{2}$ above are independent of $k$ and $\lambda$.

It is clear from the remarks above that Theorem 1.2 will be an immediate consequence of Theorem 2.1. We present the proof of Theorem $2.1 \mathrm{in} \S 4$.

\section{Asymptotic properties of Laguerre functions}

The two asymptotic formulae below hold uniformly in their respective ranges of validity (which overlap) and are due to Erdélyi [3]. In what follows, $\nu=4 k+2 \delta+2$ and $N=\frac{1}{4} \nu$.

\subsection{The Bessel asymptotic forms}

Let $0 \leqslant x \leqslant b \nu, b<1$. Then, for $k \geqslant k_{0}$,

$$
\begin{aligned}
\Lambda_{k}^{\delta}(x)=\left(\frac{(\delta+k) !}{k !}\right)^{1 / 2} & 2^{\delta-1 / 2} \nu^{-\delta / 2} \\
& \times\left(\frac{\nu}{x}\right)^{1 / 2}\left(\frac{\psi}{\psi^{\prime}}\right)^{1 / 2}\left\{J_{\delta}(\nu \psi)+O\left[\nu^{-1}\left(\frac{x}{\nu-x}\right)^{1 / 2} \tilde{J}_{\delta}(\nu \psi)\right]\right\},
\end{aligned}
$$

and so

$$
\Lambda_{k}^{\delta}(x)=C_{1}(\delta)\left(\frac{\nu}{x}\right)^{1 / 2}\left(\frac{\psi}{\psi^{\prime}}\right)^{1 / 2}\left\{J_{\delta}(\nu \psi)+O\left[\nu^{-1}\left(\frac{x}{\nu-x}\right)^{1 / 2} \tilde{J}_{\delta}(\nu \psi)\right]\right\},
$$

where $C_{1}(\delta)$ is a constant independent of $k, \psi=\psi(t)$ satisfies

$$
\psi^{\prime}(t)=\frac{1}{2}\left(\frac{1}{t}-1\right)^{1 / 2}
$$

and $t=x / \nu$. For $0 \leqslant t<1$,

$$
\psi(t)=\frac{1}{2}\left[\left(t-t^{2}\right)^{1 / 2}+\sin ^{-1} t^{1 / 2}\right],
$$

and

$$
\tilde{J}_{\delta}(u)= \begin{cases}J_{\delta}(u) & \text { if } u \text { is sufficiently small } \\ \left(\left|J_{\delta}(u)\right|^{2}+\left|Y_{\delta}(u)\right|^{2}\right)^{1 / 2} & \text { otherwise. }\end{cases}
$$

Here $Y_{\delta}$ and $J_{\delta}$ are Bessel functions of order $\delta$. 
Lemma 3.1. If $0 \leqslant t \leqslant \frac{1}{2}$, then $\frac{1}{2} t^{1 / 2} \leqslant \psi(t) \leqslant t^{1 / 2}$.

Proof. Let $f(t)=\left(t-t^{2}\right)^{1 / 2}+\sin ^{-1} t^{1 / 2}$. Note then that

$$
f^{\prime}(t)=\left(\frac{1-t}{t}\right)^{1 / 2}
$$

Now, if $0 \leqslant s \leqslant \frac{1}{2}$, we have $\frac{1}{2} s^{-1 / 2} \leqslant f^{\prime}(s) \leqslant s^{-1 / 2}$, and so

$$
\frac{1}{2} \int_{0}^{t} s^{-1 / 2} \mathrm{~d} s \leqslant \int_{0}^{t} f^{\prime}(s) \mathrm{d} s \leqslant \int_{0}^{t} s^{-1 / 2} \mathrm{~d} s,
$$

which implies that $t^{1 / 2} \leqslant f(t) \leqslant 2 t^{1 / 2}$, since $f(0)=0$.

\subsection{The Airy asymptotic forms}

Let $0<a \nu \leqslant x, a>0$. Then, for $k \geqslant k_{0}$,

$$
\begin{aligned}
\Lambda_{k}^{\delta}(x)=\frac{(-1)^{k}}{(k !(\delta+k) !)^{1 / 2}} 2^{5 / 6} N^{N+1 / 6} \mathrm{e}^{-N} x^{-1 / 2} & \\
& \times\left(\frac{\pi}{-\phi^{\prime}}\right)^{1 / 2}\left\{\operatorname{Ai}\left(-\nu^{2 / 3} \phi\right)+O\left[x^{-1} \widetilde{\operatorname{Ai}}\left(-\nu^{2 / 3} \phi\right)\right]\right\},
\end{aligned}
$$

and so, using Stirling's formula,

$$
\Lambda_{k}^{\delta}(x)=C_{2}(\delta)(-1)^{k} \nu^{1 / 6} x^{-1 / 2}\left(\frac{1}{-\phi^{\prime}}\right)^{1 / 2}\left\{\operatorname{Ai}\left(-\nu^{2 / 3} \phi\right)+O\left[x^{-1} \widetilde{\operatorname{Ai}}\left(-\nu^{2 / 3} \phi\right)\right]\right\}
$$

where $C_{2}(\delta)$ is a constant independent of $k, \phi=\phi(t)$ satisfies

$$
[\phi(t)]^{1 / 2} \phi^{\prime}(t)=\frac{1}{2}\left(\frac{1}{t}-1\right)^{1 / 2}
$$

and again $t=x / \nu$. Now we can show that

$$
\phi(t)=\left(\frac{3}{4}\right)^{2 / 3} \begin{cases}{\left[\cos ^{-1} t^{1 / 2}-\left(t-t^{2}\right)^{1 / 2}\right]^{2 / 3}} & \text { if } 0<t \leqslant 1 \\ -\left[\left(t-t^{2}\right)^{1 / 2}-\cosh ^{-1} t^{1 / 2}\right]^{2 / 3} & \text { if } t>1\end{cases}
$$

and

$$
\widetilde{\operatorname{Ai}}(z)= \begin{cases}\operatorname{Ai}(z) & \text { if } z \geqslant 0, \\ \left(|\operatorname{Ai}(z)|^{2}+|\operatorname{Bi}(z)|^{2}\right)^{1 / 2} & \text { if } z<0 .\end{cases}
$$

Here Ai and Bi are Airy integrals.*

* $\operatorname{Ai}(z)$ and $\operatorname{Bi}(z)$ are independent solutions of the differential equation $\mathrm{d}^{2} y / \mathrm{d} z^{2}=z y$ and have the integral representations

$$
\operatorname{Ai}(z)=\frac{1}{\pi} \int_{0}^{\infty} \cos \left(\frac{1}{3} t^{3}+z t\right) \mathrm{d} t
$$

and

$$
\operatorname{Bi}(z)=\frac{1}{\pi} \int_{0}^{\infty}\left\{\mathrm{e}^{\left(t^{3}+z t\right) / 3}+\sin \left(\frac{1}{3} t^{3}+z t\right)\right\} \mathrm{d} t
$$


Lemma 3.2. If $\frac{1}{2} \leqslant t \leqslant 1$, then $\frac{1}{2}(1-t) \leqslant \phi(t) \leqslant 1-t$.

Proof. Let $g(t)=\cos ^{-1} t^{1 / 2}-\left(t-t^{2}\right)^{1 / 2}$. Note then that $g^{\prime}(t)=-(1-t / t)^{1 / 2}$. Now if $\frac{1}{2} \leqslant s \leqslant 1$, we have $(1-s)^{1 / 2} \leqslant-g^{\prime}(s) \leqslant 2(1-s)^{1 / 2}$, and so

$$
\int_{t}^{1}(1-s)^{1 / 2} \mathrm{~d} s \leqslant-\int_{t}^{1} g^{\prime}(s) \mathrm{d} s \leqslant 2 \int_{t}^{1}(1-s)^{1 / 2} \mathrm{~d} s
$$

which implies that $\frac{2}{3}(1-t)^{3 / 2} \leqslant g(t) \leqslant \frac{4}{3}(1-t)^{3 / 2}$, since $g(1)=0$.

Note also that, for $z>0$,

$$
\begin{aligned}
& \operatorname{Ai}(-z)=\frac{1}{3} z^{1 / 2}\left[J_{1 / 3}\left(\frac{2}{3} z^{3 / 2}\right)+J_{-1 / 3}\left(\frac{2}{3} z^{3 / 2}\right)\right] \\
& \operatorname{Bi}(-z)=\left(\frac{1}{3} z\right)^{1 / 2}\left[J_{1 / 3}\left(\frac{2}{3} z^{3 / 2}\right)+J_{-1 / 3}\left(\frac{2}{3} z^{3 / 2}\right)\right] .
\end{aligned}
$$

\subsection{Bessel functions}

The Bessel functions, defined for real $k>-\frac{1}{2}$ by the formula

$$
J_{k}(\lambda)=\frac{1}{\pi^{1 / 2} \Gamma\left(k+\frac{1}{2}\right)} \frac{1}{2} \lambda^{k} \int_{-1}^{1} \mathrm{e}^{\mathrm{i} \lambda t}\left(1-t^{2}\right)^{k-1 / 2} \mathrm{~d} t,
$$

are a model case for oscillatory integrals in one dimension, and using this theory we can show that

$$
J_{k}(\lambda)=\sigma_{1}(\lambda) \mathrm{e}^{\mathrm{i} \lambda}+\sigma_{2}(\lambda) \mathrm{e}^{-\mathrm{i} \lambda},
$$

where $\left|\sigma_{i}^{(\ell)}(\lambda)\right| \leqslant c_{\ell}(1+\lambda)^{-1 / 2-\ell}$ (see, for example, [13]).

\subsection{Trivial estimates}

It follows from the asymptotics above that for $k$ large we have the following crude estimates for our Laguerre function (see $[\mathbf{1}]$ ):

$$
\left|\Lambda_{k}^{\delta}(x)\right| \leqslant C \begin{cases}(x \nu)^{\delta / 2} & \text { if } 0 \leqslant x \leqslant 1 / \nu, \\ (x \nu)^{-1 / 4} & \text { if } 1 / \nu \leqslant x \leqslant \frac{1}{2} \nu, \\ \nu^{-1 / 4}(\nu-x)^{-1 / 4} & \text { if } \frac{1}{2} \nu \leqslant x \leqslant \nu-\nu^{1 / 3}, \\ \nu^{-1 / 3} & \text { if } \nu-\nu^{1 / 3} \leqslant x \leqslant \nu+\nu^{1 / 3}, \\ \nu^{-1 / 4}(x-\nu)^{-1 / 4} \mathrm{e}^{-\gamma_{1} \nu^{-1 / 2}(x-\nu)^{3 / 2}} & \text { if } \nu+\nu^{1 / 3} \leqslant x \leqslant \frac{3}{2} \nu, \\ \mathrm{e}^{-\gamma_{2} x} & \text { if } x \geqslant \frac{3}{2} \nu,\end{cases}
$$

where $\gamma_{1}, \gamma_{2}>0$ are fixed constants. 


\section{Proof of Theorem 2.1}

It is natural to consider the cases for bounded and unbounded $k$ separately. We now fix $k_{0}$ to be a large constant and note that, for $k \leqslant k_{0}$, we can easily verify, by integration by parts, that

$$
|\mu(k, \lambda)| \leqslant C(1+|\lambda|)^{-N},
$$

for all $N \geqslant 0$.

Since we are now only interested in the case when $k$ is large, our main object of study, namely $\mu(k, \lambda)$, is therefore essentially

$$
\mathcal{I}=\int_{0}^{\infty} \mathrm{e}^{-\varepsilon r^{-\beta}} \chi(r) r^{-1-\alpha} \mathrm{e}^{\mathrm{i} r^{-\beta}}(x \nu)^{(1-n) / 2} \Lambda_{k}^{n-1}(x) \mathrm{d} r,
$$

where $x=\frac{1}{2}|\lambda| r^{2}$. We can of course always integrate by parts in $r$, but we must take care of what happens when the derivative is applied to the amplitude of $\mathcal{I}$.

Recall that $\Lambda_{k}^{\delta}(x)=c_{k}^{\delta} L_{k}^{\delta}(x) \mathrm{e}^{-x / 2} x^{\delta / 2}$. Now, since

$$
\frac{\mathrm{d}}{\mathrm{d} x} L_{k}^{\delta}(x)=-L_{k-1}^{\delta+1}(x) \quad \text { and } \quad c_{k-1}^{\delta+1}=k^{-1 / 2} c_{k}^{\delta},
$$

it follows that

$$
\frac{\mathrm{d}}{\mathrm{d} x} \Lambda_{k}^{\delta}(x)=\frac{1}{2}\left(\frac{\delta}{x}-1\right) \Lambda_{k}^{\delta}(x)-\left(\frac{k}{x}\right)^{1 / 2} \Lambda_{k-1}^{\delta+1}(x) .
$$

Therefore, using the fact that $\partial_{r} x=2 x / r$, we see that

$$
\partial_{r} \Lambda_{k}^{\delta}(x)=-r^{-1}\left[(x-\delta) \Lambda_{k}^{\delta}(x)+2(x k)^{1 / 2} \Lambda_{k-1}^{\delta+1}(x)\right] .
$$

If we instead take $N$ derivatives, it is easy to see that

$\partial_{r}^{N} \Lambda_{k}^{\delta}(x)=r^{-N}\left[P_{N}(x) \Lambda_{k}^{\delta}(x)+(x k)^{1 / 2} P_{N-1}(x) \Lambda_{k-1}^{\delta+1}(x)+\cdots+(x k)^{N / 2} P_{0}(x) \Lambda_{k-N}^{\delta+N}(x)\right]$,

that is,

$$
\partial_{r}^{N} \Lambda_{k}^{\delta}(x)=r^{-N} \sum_{\ell=0}^{N}(x k)^{\ell / 2} P_{N-\ell}(x) \Lambda_{k-\ell}^{\delta+\ell}(x),
$$

where $P_{N-\ell}(x)$ is some polynomial of degree $N-\ell$ in $x$.

We therefore see that integration by parts will, in general, only help us if

$$
\max \left\{(x \nu)^{1 / 2}, x\right\} \leqslant C_{1} r^{-\beta} .
$$

In order to estimate $\mathcal{I}$ we will make use of the asymptotics for Laguerre functions presented in $\S 3$. It is then natural to consider six separate regions and write $\mathcal{I}=\mathcal{I}_{1}+\cdots+\mathcal{I}_{6}$, where

$$
\mathcal{I}_{j}=\int_{0}^{\infty} \chi_{j}^{\varepsilon}(r) r^{-1-\alpha} \mathrm{e}^{\mathrm{i} r^{-\beta}}(x \nu)^{(1-n) / 2} \Lambda_{k}^{n-1}(x) \mathrm{d} r
$$

$\chi_{1}^{\varepsilon}(r)=\mathrm{e}^{-\varepsilon r^{-\beta}} \chi(r) \vartheta_{j}(x, \nu)$, and each $\vartheta_{j}(x, \nu)$ localizes smoothly to the $j$ th interval indicated in $\S 3.4$. 


\subsection{Neighbourhood of $0: 0 \leqslant x \leqslant 1 / \nu$}

Note that here $x \nu \leqslant 1$ and $\left|\Lambda_{k}^{\delta}(x)\right| \leqslant C(x \nu)^{\delta / 2}$. Using our trivial estimate it is easy to see that

$$
\left|\partial_{r}^{N} \Lambda_{k}^{\delta}(x)\right| \leqslant C r^{-N}(x \nu)^{(\delta / 2)+N} .
$$

Therefore, by integrating by parts $N$ times, we obtain the estimate

$$
\begin{aligned}
\left|\mathcal{I}_{1}\right| & =\left|\int_{0}^{\infty} \chi_{1}^{\varepsilon}(r) r^{-1-\alpha} \mathrm{e}^{\mathrm{i} r^{-\beta}}(x \nu)^{(1-n) / 2} \Lambda_{k}^{n-1}(x) \mathrm{d} r\right| \\
& \leqslant C \int_{r \leqslant \min \left\{1,(|\lambda| \nu)^{-1 / 2}\right\}} r^{-1-\alpha+N \beta} \mathrm{d} r \\
& \leqslant C(|\lambda| \nu)^{(\alpha-N \beta) / 2},
\end{aligned}
$$

for any $N \geqslant 0$.

\subsection{Oscillatory interval I: $1 / \nu \leqslant x \leqslant \nu / 2$}

Note that here $\frac{1}{2} \nu \leqslant \nu-x<\nu$ and $\left|\Lambda_{k}^{\delta}(x)\right| \leqslant C(x \nu)^{-1 / 4}$.

In this interval we will make explicit use of the oscillation in the main term of our asymptotic expansion, which in this case, is given in terms of Bessel functions (see § 3.1). We note here that from (3.2) and Lemma 3.1 it follows that

$$
\psi^{\prime} \sim\left(\frac{\nu}{x}\right)^{1 / 2}, \quad \psi \sim\left(\frac{x}{\nu}\right)^{1 / 2} .
$$

The following estimates are then immediate:

$$
\begin{gathered}
(\nu \psi)^{-1 / 2} \sim(x \nu)^{-1 / 4} \quad \text { and } \quad \partial_{r}\left[(\nu \psi)^{-1 / 2}\right]=-\frac{x}{r \nu^{3 / 2}} \psi^{-3 / 2} \psi^{\prime} \sim-\frac{1}{r}(x \nu)^{-1 / 4}, \\
\partial_{r} \nu \psi=\frac{1}{r} x^{1 / 2}(\nu-x)^{1 / 2} \sim \frac{1}{r}(x \nu)^{1 / 2} \quad \text { and } \quad \partial_{r}^{2} \nu \psi=-\frac{1}{r^{2}} \frac{x^{3 / 2}}{(\nu-x)^{1 / 2}} \sim \frac{1}{r^{2}} x^{3 / 2} \nu^{-1 / 2}, \\
\left(\frac{\nu}{x}\right)^{1 / 2}\left(\frac{\psi}{\psi^{\prime}}\right)^{1 / 2} \leqslant C \quad \text { and }\left|\partial_{r}\left[\left(\frac{\nu}{x}\right)^{1 / 2}\left(\frac{\psi}{\psi^{\prime}}\right)^{1 / 2}\right]\right| \leqslant C \frac{1}{r} .
\end{gathered}
$$

Case 1. $(x \nu)^{1 / 2} \leqslant C_{1} r^{-\beta}$. Since we can integrate by parts $N$ times and $x \leqslant(x \nu)^{1 / 2}$, it suffices to estimate

$$
I_{2}=\int_{0}^{\infty} \chi_{2}^{\varepsilon}(r) r^{-1-\alpha+N \beta} \mathrm{e}^{\mathrm{i} r^{-\beta}}(x \nu)^{(N+1-n) / 2} \Lambda_{k-N}^{n-1+N}(x) \mathrm{d} r .
$$

Using the Bessel asymptotic forms (3.1), we may write $I_{2}=c B+E_{B}$, where

$$
B=\int_{0}^{\infty} \chi_{2}^{\varepsilon}(r) r^{-1-\alpha+N \beta} \mathrm{e}^{\mathrm{i} r^{-\beta}}(x \nu)^{(N+1-n) / 2}\left(\frac{\nu}{x}\right)^{1 / 2}\left(\frac{\psi}{\psi^{\prime}}\right)^{1 / 2} J_{n-1+N}(\nu \psi) \mathrm{d} r .
$$


Error term:

$$
\begin{aligned}
\left|E_{B}\right| \leqslant & C \int_{0}^{\infty} \chi_{2}^{\varepsilon}(r) r^{-1-\alpha+N \beta}(x \nu)^{(N+1-n) / 2} \\
& \times\left(\frac{\nu}{x}\right)^{1 / 2}\left(\frac{\psi}{\psi^{\prime}}\right)^{1 / 2} \nu^{-1}\left(\frac{x}{\nu-x}\right)^{1 / 2}\left|\tilde{J}_{n-1+N}(\nu \psi)\right| \mathrm{d} r \\
\leqslant & C \int_{0}^{\infty} \chi_{2}^{\varepsilon}(r) r^{-1-\alpha+N \beta}(x \nu)^{(N-n-1 / 2) / 2} \mathrm{~d} r \\
\leqslant & C(|\lambda| \nu)^{(N-n-1 / 2) / 2} \int_{r^{\beta+1} \leqslant(|\lambda| \nu)^{-1 / 2}} r^{-1-\alpha+N(\beta+1)-(n+1 / 2)} \mathrm{d} r \\
\leqslant & C(|\lambda| \nu)^{(\alpha-(n+1 / 2) \beta) / 2(\beta+1)},
\end{aligned}
$$

provided $N$ is chosen to be sufficiently large.

Main term: since $\nu \psi \geqslant(x \nu)^{1 / 2} \geqslant 1$, it follows from (3.5) that

$$
J_{n-1+N}(\nu \psi)=\sigma_{1}(\nu \psi) \mathrm{e}^{\mathrm{i} \nu \psi}+\sigma_{2}(\nu \psi) \mathrm{e}^{-\mathrm{i} \nu \psi},
$$

where each $\sigma_{i}$ is a symbol of order $-\frac{1}{2}$. Since $\partial_{r} \psi \sim \psi / r$, it follows that

$$
\left|\partial_{r}^{\ell} \sigma_{i}(\nu \psi)\right| \leqslant C r^{-\ell}(\nu \psi)^{-1 / 2}
$$

Write $B=B_{1}+B_{2}$, where

$$
\begin{aligned}
& B_{1}=\int_{0}^{\infty} \chi_{2}^{\varepsilon}(r) r^{-1-\alpha+N \beta} \mathrm{e}^{\mathrm{i}\left[r^{-\beta}+\nu \psi\right]}(x \nu)^{(N+1-n) / 2}\left(\frac{\nu}{x}\right)^{1 / 2}\left(\frac{\psi}{\psi^{\prime}}\right)^{1 / 2} \sigma_{1}(\nu \psi) \mathrm{d} r \\
& B_{2}=\int_{0}^{\infty} \chi_{2}^{\varepsilon}(r) r^{-1-\alpha+N \beta} \mathrm{e}^{\mathrm{i}\left[r^{-\beta}+\nu \psi\right]}(x \nu)^{(N+1-n) / 2}\left(\frac{\nu}{x}\right)^{1 / 2}\left(\frac{\psi}{\psi^{\prime}}\right)^{1 / 2} \sigma_{2}(\nu \psi) \mathrm{d} r .
\end{aligned}
$$

Let us first consider the integral $B_{1}$, and let $\varphi(r)=r^{-\beta}+\nu \psi$. Now

$$
\partial_{r} \varphi(r)=-\beta r^{-(\beta+1)}+\frac{1}{r} x^{1 / 2}(\nu-x)^{1 / 2},
$$

and so, if $C_{1}$ is chosen to be sufficiently small, it follows that

$$
\left|\partial_{r} \varphi(r)\right| \geqslant C r^{-(\beta+1)} .
$$

In addition to this we also have

$$
\partial_{r}^{2} \varphi(r)=\beta(\beta+1) r^{-(\beta+2)}-\frac{1}{r^{2}} \frac{x^{3 / 2}}{(\nu-x)^{1 / 2}} \geqslant C r^{-(\beta+2)} .
$$

If we let

$$
a(r)=\chi_{2}^{\varepsilon}(r) r^{-1-\alpha+N \beta}(x \nu)^{(N+1-n) / 2}\left(\frac{\nu}{x}\right)^{1 / 2}\left(\frac{\psi}{\psi^{\prime}}\right)^{1 / 2} \sigma_{1}(\nu \psi)
$$


then, for all $\ell=0,1, \ldots$, we have

$$
\left|\partial_{r}^{\ell} a(r)\right| \leqslant C r^{-1-\alpha+N \beta-\ell}(x \nu)^{N / 2}(\nu \psi)^{-1 / 2} .
$$

Applying van der Corput's lemma (integration by parts) gives

$$
\begin{aligned}
\left|B_{1}\right| & \leqslant C \int_{r^{\beta+1} \leqslant(|\lambda| \nu)^{-1 / 2}} r^{-1-\alpha+(N+1) \beta}(x \nu)^{(2 N+1-2 n) / 4} \mathrm{~d} r \\
& \leqslant C(|\lambda| \nu)^{(\alpha-(n+1 / 2) \beta) / 2(\beta+1)}
\end{aligned}
$$

provided $N$ is chosen to be sufficiently large, as before.

Of course the phase in $B_{2}$ is never stationary, so we obtain the same estimate for $B_{2}$.

Case 2. $(x \nu)^{1 / 2} \geqslant C_{1} r^{-\beta}$. Here we will not integrate by parts, so it suffices to estimate

$$
\mathcal{I}_{2}=\int_{0}^{\infty} \chi_{2}^{\varepsilon}(r) r^{-1-\alpha} \mathrm{e}^{\mathrm{i} r^{-\beta}}(x \nu)^{(1-n) / 2} \Lambda_{k}^{n-1}(x) \mathrm{d} r
$$

Using the asymptotic forms (3.1) we may write $\mathcal{I}_{2}=c \mathcal{B}+\mathcal{E}_{B}$, where

$$
\mathcal{B}=\int_{0}^{\infty} \chi_{2}^{\varepsilon}(r) r^{-1-\alpha} \mathrm{e}^{\mathrm{i} r^{-\beta}}(x \nu)^{(1-n) / 2}\left(\frac{\nu}{x}\right)^{1 / 2}\left(\frac{\psi}{\psi^{\prime}}\right)^{1 / 2} J_{n-1}(\nu \psi) \mathrm{d} r .
$$

Error term:

$$
\begin{aligned}
\left|\mathcal{E}_{B}\right| & \leqslant C \int_{0}^{\infty} \chi_{2}^{\varepsilon}(r) r^{-1-\alpha}(x \nu)^{(1-n) / 2}\left(\frac{\nu}{x}\right)^{1 / 2}\left(\frac{\psi}{\psi^{\prime}}\right)^{1 / 2} \nu^{-1}\left(\frac{x}{\nu-x}\right)^{1 / 2}\left|\tilde{J}_{n-1}(\nu \psi)\right| \mathrm{d} r \\
& \leqslant C \int_{0}^{\infty} \chi_{2}^{\varepsilon}(r) r^{-1-\alpha}(x \nu)^{-(n+1 / 2) / 2} \mathrm{~d} r \\
& \leqslant C(|\lambda| \nu)^{-(n+1 / 2) / 2} \int_{r^{\beta+1} \geqslant(|\lambda| \nu)^{-1 / 2}} r^{-1-\alpha-(n+1 / 2)} \mathrm{d} r \\
& \leqslant C(|\lambda| \nu)^{(\alpha-(n+1 / 2) \beta) / 2(\beta+1)} .
\end{aligned}
$$

Main term: as above we will use (3.5) and write $\mathcal{B}=\mathcal{B}_{1}+\mathcal{B}_{2}$, where

$$
\begin{aligned}
& \mathcal{B}_{1}=\int_{0}^{\infty} \chi_{2}^{\varepsilon}(r) r^{-1-\alpha} \mathrm{e}^{\mathrm{i}\left[r^{-\beta}+\nu \psi\right]}\left(\frac{\nu}{x}\right)^{1 / 2}(x \nu)^{(1-n) / 2}\left(\frac{\psi}{\psi^{\prime}}\right)^{1 / 2} \sigma_{1}(\nu \psi) \mathrm{d} r, \\
& \mathcal{B}_{2}=\int_{0}^{\infty} \chi_{2}^{\varepsilon}(r) r^{-1-\alpha} \mathrm{e}^{\mathrm{i}\left[r^{-\beta}-\nu \psi\right]}\left(\frac{\nu}{x}\right)^{1 / 2}(x \nu)^{(1-n) / 2}\left(\frac{\psi}{\psi^{\prime}}\right)^{1 / 2} \sigma_{2}(\nu \psi) \mathrm{d} r .
\end{aligned}
$$

Let us first consider the integral $\mathcal{B}_{1}$, and again let $\varphi(r)=r^{-\beta}+\nu \psi$. Recall that

$$
\partial_{r} \varphi(r)=-\beta r^{-(\beta+1)}+\frac{1}{r} x^{1 / 2}(\nu-x)^{1 / 2},
$$

and so, if we were to choose a constant $C_{2}$ sufficiently large, it would follow that

$$
\left|\partial_{r} \varphi(r)\right| \geqslant C \frac{1}{r} x^{1 / 2}(\nu-x)^{1 / 2}
$$


whenever $(x \nu)^{1 / 2} \geqslant C_{2} r^{-\beta}$, and hence, as before,

$$
\begin{aligned}
\left|\mathcal{B}_{1}\right| & \leqslant C \int_{r^{\beta+1} \geqslant(|\lambda| \nu)^{-1 / 2}} r^{-1-\alpha}(x \nu)^{-(2 n+1) / 4} \mathrm{~d} r \\
& \leqslant C(|\lambda| \nu)^{(\alpha-(n+1 / 2) \beta) / 2(\beta+1)} .
\end{aligned}
$$

Of course the phase in $\mathcal{B}_{2}$ is trivially never critical in the complete range, so we obtain the same estimate for $\mathcal{B}_{2}$ everywhere.

We are thus left to estimate $\mathcal{B}_{1}$ when $C_{1} r^{-\beta} \leqslant(x \nu)^{1 / 2} \leqslant C_{2} r^{-\beta}$, which means $r \sim$ $(|\lambda| \nu)^{-1 / 2(\beta+1)}$. Now making the change of variables $r=s(|\lambda| \nu)^{-1 / 2(\beta+1)}$, we see that

$$
\begin{aligned}
\mathcal{B}_{1}=(|\lambda| \nu)^{\alpha / 2(\beta+1)} & \\
& \times \int_{0}^{\infty} \vartheta(s) s^{-1-\alpha} \exp \left\{\mathrm{i}(|\lambda| \nu)^{\beta / 2(\beta+1)} \Phi(s)\right\}\left(\frac{\nu}{x}\right)^{1 / 2}(x \nu)^{(1-n) / 2}\left(\frac{\psi}{\psi^{\prime}}\right)^{1 / 2} \sigma_{1}(\nu \psi) \mathrm{d} r,
\end{aligned}
$$

where $\vartheta$ is smooth and supported where $s \sim 1$ and $\Phi(s)=s^{-\beta}+(|\lambda| \nu)^{-\beta / 2(\beta+1)} \nu \psi$.

Although our phase $\Phi(s)$ may now be stationary in this range we do have the following.

Lemma 4.1. If $s \sim 1$ and $x \leqslant \frac{1}{2} \nu$, then $\left|\partial_{s} \Phi(s)\right|+\left|\partial_{s}^{2} \Phi(s)\right| \geqslant C_{0}>0$.

Proof. Recall that

$$
\partial_{s} \Phi(s)=-\beta s^{-(\beta+1)}+(|\lambda| \nu)^{-\beta / 2(\beta+1)} \frac{1}{s} x^{1 / 2}(\nu-x)^{1 / 2},
$$

and

$$
\partial_{s}^{2} \Phi(s)=\beta(\beta+1) s^{-(\beta+2)}-(|\lambda| \nu)^{-\beta / 2(\beta+1)} \frac{1}{s^{2}} \frac{x^{3 / 2}}{(\nu-x)^{1 / 2}} .
$$

Hence $\partial_{s} \Phi(s)=0$ if and only if

$$
(|\lambda| \nu)^{-\beta / 2(\beta+1)} \frac{1}{s} x^{1 / 2}(\nu-x)^{1 / 2}=\beta s^{-(\beta+1)} .
$$

It is therefore clear that if

$$
(|\lambda| \nu)^{-\beta / 2(\beta+1)} \frac{1}{s} x^{1 / 2}(\nu-x)^{1 / 2} \geqslant\left(1+\frac{1}{2} \beta\right) \beta s^{-(\beta+1)},
$$

then

$$
\left|\partial_{s} \Phi(s)\right| \geqslant C s^{-(\beta+1)},
$$

while if

$$
(|\lambda| \nu)^{-\beta / 2(\beta+1)} \frac{1}{s} x^{1 / 2}(\nu-x)^{1 / 2} \leqslant\left(1+\frac{1}{2} \beta\right) \beta s^{-(\beta+1)},
$$

then we have

$$
\partial_{s}^{2} \Phi(s)=\beta(\beta+1) s^{-(\beta+2)}-\frac{1}{s} \frac{x}{\nu-x}(|\lambda| \nu)^{-\beta / 2(\beta+1)} \frac{1}{s} x^{1 / 2}(\nu-x)^{1 / 2} \geqslant C s^{-(\beta+2)},
$$

since $0<x /(\nu-x) \leqslant 1$. 
We note that, since

$$
-\partial_{s}^{3} \Phi(s)=\beta(\beta+1)(\beta+2) s^{-(\beta+3)}+(|\lambda| \nu)^{-\beta / 2(\beta+1)} \frac{\nu}{s^{3}} \frac{x^{3 / 2}}{(\nu-x)^{3 / 2}}>0,
$$

our phase $\Phi$ can have at most two separated critical points; this follows from the fact that, for all $\ell=0,1, \ldots$, we have $\left|\partial_{s}^{\ell} \Phi(s)\right| \leqslant c_{\ell}$. In addition to this we note that if we set

$$
a(s)=\vartheta(s) s^{-1-\alpha}(x \nu)^{(1-n) / 2}\left(\frac{\nu}{x}\right)^{1 / 2}\left(\frac{\psi}{\psi^{\prime}}\right)^{1 / 2} \sigma_{1}(\nu \psi),
$$

then, for all $\ell=0,1, \ldots$,

$$
\left|\partial_{s}^{\ell} a(s)\right| \leqslant C(x \nu)^{(1-n) / 2-(1 / 4)} \sim(|\lambda| \nu)^{-(n-(1 / 2)) \beta / 2(\beta+1)} .
$$

Applying van der Corput's lemma therefore gives

$$
\left|\mathcal{B}_{1}\right| \leqslant C(|\lambda| \nu)^{(\alpha-n \beta) / 2(\beta+1)} .
$$

We have therefore established that $|\mathcal{B}| \leqslant C(|\lambda| \nu)^{(\alpha-n \beta) / 2(\beta+1)}$, and hence the same estimate for $\mathcal{I}_{2}$.

\subsection{Oscillatory interval II: $\frac{1}{2} \nu \leqslant x \leqslant \nu-\nu^{1 / 3}$}

We now have $\nu^{1 / 3} \leqslant \nu-x \leqslant \frac{1}{2} \nu$ and the trivial estimate $\left|\Lambda_{k}^{\delta}(x)\right| \leqslant C x^{-1 / 4}(\nu-x)^{-1 / 4}$.

The situation here is much the same as it was in $\S 4.2$, but here we must instead use the Airy asymptotic form. In order to do better than the trivial estimate, we will again make use the oscillation in the main term of our asymptotic expansion. It follows from Lemma 3.2 that

$$
\phi \sim \frac{\nu-x}{\nu} .
$$

From this and (3.4) it is immediately clear that

$$
\frac{1}{10} \leqslant \phi^{\prime} \leqslant 10 \text { and } \quad \phi^{\prime \prime} \leqslant C
$$

Case 1. $(x \nu)^{1 / 2} \leqslant C_{1} r^{-\beta}$. We must integrate by parts and, since $x \leqslant(x \nu)^{1 / 2}$, it suffices to estimate

$$
I_{3}=\int_{0}^{\infty} \chi_{3}^{\varepsilon}(r) r^{-1-\alpha+N \beta} \mathrm{e}^{\mathrm{i} r^{-\beta}}(x \nu)^{(N+1-n) / 2} \Lambda_{k-N}^{n-1+N}(x) \mathrm{d} r .
$$

Using the Airy asymptotic forms (3.3) we may write this as $I_{3}=c A+E_{A}$, where

$$
A=\int_{0}^{\infty} \chi_{3}^{\varepsilon}(r) r^{-1-\alpha+N \beta} \mathrm{e}^{\mathrm{i} r^{-\beta}}(x \nu)^{(N+1-n) / 2} x^{-1 / 2} \nu^{1 / 6}\left(\frac{1}{-\phi^{\prime}}\right)^{1 / 2} \operatorname{Ai}\left(-\nu^{2 / 3} \phi\right) \mathrm{d} r .
$$


Error term:

$$
\begin{aligned}
\left|E_{A}\right| & \leqslant \int_{0}^{\infty} \chi_{3}^{\varepsilon}(r)(r) r^{-1-\alpha+N \beta}(x \nu)^{(N+1-n) / 2} x^{-3 / 2} \nu^{1 / 6}\left(\frac{1}{-\phi^{\prime}}\right)^{1 / 2}\left|\widetilde{\operatorname{Ai}}\left(-\nu^{2 / 3} \phi\right)\right| \mathrm{d} r \\
& \leqslant C \int_{0}^{\infty} \chi_{3}^{\varepsilon}(r)(r) r^{-1-\alpha+N \beta}(x \nu)^{(N-(1 / 2)-n) / 2} \nu^{1 / 4}(\nu-x)^{-1 / 4} \mathrm{~d} r \\
& \leqslant C(|\lambda| \nu)^{(\alpha-(n+1 / 2) \beta) / 2(\beta+1)} \nu^{-3 / 4} \int_{\nu-x \leqslant \nu}(\nu-x)^{-1 / 4} \mathrm{~d} x \\
& \leqslant C(|\lambda| \nu)^{(\alpha-(n+1 / 2) \beta) / 2(\beta+1)}
\end{aligned}
$$

provided that $N$ is chosen to be sufficiently large.

Main term: recall that, for $z>0$,

$$
\operatorname{Ai}(-z)=\frac{1}{3} z^{1 / 2}\left[J_{1 / 3}\left(\frac{2}{3} z^{3 / 2}\right)+J_{-1 / 3}\left(\frac{2}{3} z^{3 / 2}\right)\right],
$$

and, since $J_{1 / 3}$ and $J_{-1 / 3}$ satisfy the same bounds for large $z$, it suffices to estimate

$$
\begin{aligned}
\tilde{A}=\int_{0}^{\infty} \chi_{3}^{\varepsilon}(r) r^{-1-\alpha+N \beta} \mathrm{e}^{\mathrm{i} r^{-\beta}} & (x \nu)^{(N+1-n) / 2} \\
& \times x^{-1 / 2} \nu^{1 / 6}\left(\frac{1}{-\phi^{\prime}}\right)^{1 / 2}\left(\nu^{2 / 3} \phi\right)^{1 / 2} J_{1 / 3}\left(\frac{2}{3} \nu \phi^{3 / 2}\right) \mathrm{d} r .
\end{aligned}
$$

It follows from (3.5) that

$$
J_{1 / 3}\left(\frac{2}{3} \nu \phi^{3 / 2}\right)=\sigma_{1}\left(\nu \phi^{3 / 2}\right) \exp \left\{\mathrm{i} \frac{2}{3} \nu \phi^{3 / 2}\right\}+\sigma_{2}\left(\nu \phi^{3 / 2}\right) \exp \left\{-\mathrm{i} \frac{2}{3} \nu \phi^{3 / 2}\right\},
$$

where $\sigma_{i}$ is a symbol of order $-\frac{1}{2}$. We therefore write $\tilde{A}=A_{1}+A_{2}$, where

$$
\begin{aligned}
& A_{1}=\int_{0}^{\infty} \chi_{3}^{\varepsilon}(r) r^{-1-\alpha+N \beta} \exp \left\{\mathrm{i}\left[r^{-\beta}+\frac{2}{3} \nu \phi^{3 / 2}\right]\right\} \\
& \times(x \nu)^{(N+1-n) / 2} x^{-1 / 2} \nu^{1 / 6}\left(\frac{1}{-\phi^{\prime}}\right)^{1 / 2}\left(\nu^{2 / 3} \phi\right)^{1 / 2} \sigma_{1}\left(\nu \phi^{3 / 2}\right) \mathrm{d} r, \\
& A_{2}=\int_{0}^{\infty} \chi_{3}^{\varepsilon}(r) r^{-1-\alpha+}+N \beta \exp \left\{\mathrm{i}\left[r^{-\beta}-\frac{2}{3} \nu \phi^{3 / 2}\right]\right\} \\
& \times(x \nu)^{(N+1-n) / 2} x^{-1 / 2} \nu^{1 / 6}\left(\frac{1}{-\phi^{\prime}}\right)^{1 / 2}\left(\nu^{2 / 3} \phi\right)^{1 / 2} \sigma_{2}\left(\nu \phi^{3 / 2}\right) \mathrm{d} r .
\end{aligned}
$$

Let us first consider the integral $A_{1}$, and now let $\tilde{\varphi}(r)=r^{-\beta}+\frac{2}{3} \nu \phi^{3 / 2}$. We note that $\partial_{r} \tilde{\varphi}=\partial_{r} \varphi$. It therefore follows that $\tilde{\varphi}$ behaves exactly as $\varphi$ did in $\S 4.2$, and so, for $C_{1}$ chosen to be sufficiently small, we again may integrate by parts. In this case our amplitude

$$
\tilde{a}(r)=\chi_{3}^{\varepsilon}(r) r^{-1-\alpha+N \beta}(x \nu)^{(N+1-n) / 2} x^{-1 / 2} \nu^{1 / 6}\left(\frac{1}{-\phi^{\prime}}\right)^{1 / 2}\left(\nu^{2 / 3} \phi\right)^{1 / 2} \sigma_{1}\left(\nu \phi^{3 / 2}\right),
$$


courtesy of the symbol estimates $\left|\partial_{r}^{\ell} \sigma_{1}\left(\nu \phi^{3 / 2}\right)\right| \leqslant C r^{-\ell} x^{(1 / 4)+\ell}(\nu-x)^{-(3 / 4)-\ell}$, satisfies, for $\ell=0,1, \ldots$, the differential inequality

$$
\left|\partial_{r}^{\ell} \tilde{a}(r)\right| \leqslant C r^{-1-\alpha+N \beta-\ell}(\nu-x)^{-\ell-(1 / 4)} \nu^{N-n+(3 / 4)+\ell} .
$$

Integrating by parts $N^{\prime}$ times, we therefore get the estimate

$$
\begin{aligned}
\left|A_{1}\right| & \leqslant C \int_{0}^{\infty} r^{-1-\alpha+\left(N+N^{\prime}\right) \beta}(\nu-x)^{-N^{\prime}-(1 / 4)} \nu^{N+N^{\prime}-n+(3 / 4)} \mathrm{d} r \\
& \leqslant C(|\lambda| \nu)^{(\alpha-(n+1 / 4) \beta) / 2(\beta+1)} \int_{\nu^{1 / 3} \leqslant \nu-x}(\nu-x)^{-N^{\prime}-(1 / 4)} \mathrm{d} x \\
& \leqslant C(|\lambda| \nu)^{\left(\alpha-n \beta-\left(N^{\prime} / 3\right)\right) / 2(\beta+1)},
\end{aligned}
$$

again provided that $N$ is sufficiently large, and additionally that $N^{\prime} \geqslant 1$.

We of course obtain the same estimate for $A_{2}$, since its phase is trivially never stationary.

Case 2. $(x \nu)^{1 / 2} \geqslant C_{1} r^{-\beta}$. Here we will not integrate by parts first, so it suffices to estimate

$$
\mathcal{I}_{3}=\int_{0}^{\infty} \chi_{3}^{\varepsilon}(r) r^{-1-\alpha} \mathrm{e}^{\mathrm{i} r^{-\beta}}(x \nu)^{(1-n) / 2} \Lambda_{k}^{n-1}(x) \mathrm{d} r
$$

Using the asymptotic forms (3.3) we may write $\mathcal{I}_{3}=c \mathcal{A}+\mathcal{E}_{A}$, where

$$
\mathcal{A}=\int_{0}^{\infty} \chi_{3}^{\varepsilon}(r) r^{-1-\alpha} \mathrm{e}^{\mathrm{i} r^{-\beta}}(x \nu)^{(1-n) / 2} x^{-1 / 2} \nu^{1 / 6}\left(\frac{1}{-\phi^{\prime}}\right)^{1 / 2} \operatorname{Ai}\left(-\nu^{2 / 3} \phi\right) \mathrm{d} r
$$

Error term:

$$
\begin{aligned}
\left|\mathcal{E}_{A}\right| & \leqslant \int_{0}^{\infty} \chi_{3}^{\varepsilon}(r)(r) r^{-1-\alpha}(x \nu)^{(1-n) / 2} x^{-3 / 2} \nu^{1 / 6}\left(\frac{1}{-\phi^{\prime}}\right)^{1 / 2}\left|\widetilde{\mathrm{Ai}}\left(-\nu^{2 / 3} \phi\right)\right| \mathrm{d} r \\
& \leqslant C \int_{0}^{\infty} \chi_{3}^{\varepsilon}(r)(r) r^{-1-\alpha}(x \nu)^{-(n+1 / 2) / 2} \nu^{1 / 4}(\nu-x)^{-1 / 4} \mathrm{~d} r \\
& \leqslant C(|\lambda| \nu)^{(\alpha-(n+1 / 2) \beta) / 2(\beta+1)} \nu^{-3 / 4} \int_{\nu-x \leqslant \nu}(\nu-x)^{-1 / 4} \mathrm{~d} x \\
& \leqslant C(|\lambda| \nu)^{(\alpha-(n+1 / 2) \beta) / 2(\beta+1)} .
\end{aligned}
$$

Main term: as above, it suffices to consider

$$
\tilde{\mathcal{A}}=\int_{0}^{\infty} \chi_{3}^{\varepsilon}(r) r^{-1-\alpha} \mathrm{e}^{\mathrm{i} r^{-\beta}}(x \nu)^{(1-n) / 2} x^{-1 / 2} \nu^{1 / 6}\left(\frac{1}{-\phi^{\prime}}\right)^{1 / 2}\left(\nu^{2 / 3} \phi\right)^{1 / 2} J_{1 / 3}\left(\frac{2}{3} \nu \phi^{3 / 2}\right) \mathrm{d} r
$$


and as before we will write $\tilde{\mathcal{A}}=\mathcal{A}_{1}+\mathcal{A}_{2}$, where

$$
\begin{aligned}
& \mathcal{A}_{1}=\int_{0}^{\infty} \chi_{3}^{\varepsilon}(r) r^{-1-\alpha} \exp \left\{\mathrm{i}\left[r^{-\beta}+\frac{2}{3} \nu \phi^{3 / 2}\right]\right\} \\
& \times(x \nu)^{(1-n) / 2} x^{-1 / 2} \nu^{1 / 6}\left(\frac{1}{-\phi^{\prime}}\right)^{1 / 2}\left(\nu^{2 / 3} \phi\right)^{1 / 2} \sigma_{1}\left(\nu \phi^{3 / 2}\right) \mathrm{d} r, \\
& \mathcal{A}_{2}=\int_{0}^{\infty} \chi_{3}^{\varepsilon}(r) r^{-1-\alpha} \exp \left\{\mathrm{i}\left[r^{-\beta}-\frac{2}{3} \nu \phi^{3 / 2}\right]\right\} \\
& \times(x \nu)^{(1-n) / 2} x^{-1 / 2} \nu^{1 / 6}\left(\frac{1}{-\phi^{\prime}}\right)^{1 / 2}\left(\nu^{2 / 3} \phi\right)^{1 / 2} \sigma_{2}\left(\nu \phi^{3 / 2}\right) \mathrm{d} r .
\end{aligned}
$$

As before, matters reduce to the study of $\mathcal{A}_{1}$ and we again let $\tilde{\varphi}(r)=r^{-\beta}+\frac{2}{3} \nu \phi^{3 / 2}$. As in $\S 4.2$, we may choose a constant $C_{2}$ so large that

$$
\left|\partial_{r} \tilde{\varphi}(r)\right| \geqslant C \frac{1}{r} x^{1 / 2}(\nu-x)^{1 / 2}
$$

whenever $(x \nu)^{1 / 2} \geqslant C_{2} r^{-\beta}$. In this range we can therefore integrate by parts $N^{\prime}$ times and obtain

$$
\begin{aligned}
\left|\mathcal{A}_{1}\right| & \leqslant C \int_{0}^{\infty} r^{-1-\alpha}(\nu-x)^{-3 N^{\prime} / 2-1 / 4} \nu^{\left(N^{\prime} / 2\right)-n+(3 / 4)} \mathrm{d} r \\
& \leqslant C(|\lambda| \nu)^{(\alpha-n \beta) / 2(\beta+1)} \nu^{N^{\prime} / 2-1 / 4} \int_{\nu^{1 / 3} \leqslant \nu-x}(\nu-x)^{-3 N^{\prime} / 2-1 / 4} \mathrm{~d} x \\
& \leqslant C(|\lambda| \nu)^{(\alpha-n \beta) / 2(\beta+1)} .
\end{aligned}
$$

Of course the phase in $\mathcal{A}_{2}$ is trivially never critical in the complete range, so we obtain the same estimate for $\mathcal{A}_{2}$ everywhere.

We are thus left to estimate $\mathcal{A}_{1}$ when $C_{1} r^{-\beta} \leqslant(x \nu)^{1 / 2} \leqslant C_{2} r^{-\beta}$, which means that $r \sim(|\lambda| \nu)^{-1 / 2(\beta+1)}$. Making the change of variables $r=s(|\lambda| \nu)^{-1 / 2(\beta+1)}$, we see that

$$
\begin{aligned}
\mathcal{A}_{1}=(|\lambda| \nu)^{\alpha / 2(\beta+1)} \int_{0}^{\infty} \vartheta( & s) s^{-1-\alpha} \mathrm{e}^{\mathrm{i}(|\lambda| \nu)^{\beta / 2(\beta+1)} \tilde{\Phi}(s)} \\
& \times(x \nu)^{(1-n) / 2} x^{-1 / 2} \nu^{1 / 6}\left(\frac{1}{-\phi^{\prime}}\right)^{1 / 2}\left(\nu^{2 / 3} \phi\right)^{1 / 2} \sigma_{1}\left(\nu \phi^{3 / 2}\right) \mathrm{d} r
\end{aligned}
$$

where $\vartheta$ is smooth and supported where $s \sim 1$ and $\tilde{\Phi}(s)=s^{-\beta}+(|\lambda| \nu)^{-\beta / 2(\beta+1)} \nu \psi$.

We recall that $\tilde{\Phi}(s)$ satisfies the same differential inequalities as $\Phi(s)$ and thus both $\partial_{s} \tilde{\Phi}(s)$ and $\partial_{s}^{2} \tilde{\Phi}(s)$ now vanish at a point $s=s_{0}$ determined by the condition $x /(\nu-x)=$ $\beta+1$. We can, however, use the fact, noted above, that

$$
-\partial_{s}^{3} \Phi(s)=\beta(\beta+1)(\beta+2) s^{-(\beta+3)}+(|\lambda| \nu)^{-\beta / 2(\beta+1)} \frac{\nu}{s^{3}} \frac{x^{3 / 2}}{(\nu-x)^{3 / 2}}>0 .
$$


Applying the method of stationary phase (see [9, Chapter VIII, Proposition 3]) in a suitably small neighbourhood of $s_{0}$ therefore gives

$$
\begin{gathered}
\mathcal{A}_{1}=C(|\lambda| \nu)^{(\alpha-\beta / 3) / 2(\beta+1)} \nu^{-n+(1 / 2)} \exp \left\{\mathrm{i}(|\lambda| \nu)^{\beta / 2(\beta+1)} \tilde{\Phi}\left(s_{0}\right)\right\} \\
+O\left((|\lambda| \nu)^{(\alpha-2 \beta / 3) / 2(\beta+1)} \nu^{-n+(1 / 2)}\right) \\
=C(|\lambda| \nu)^{(\alpha-(n-(1 / 6)) \beta) / 2(\beta+1)} \exp \left\{\mathrm{i}(|\lambda| \nu)^{\beta / 2(\beta+1)} \tilde{\Phi}\left(s_{0}\right)\right\} \\
+O\left((|\lambda| \nu)^{(\alpha-(n+(1 / 6)) \beta) / 2(\beta+1)}\right) .
\end{gathered}
$$

Away from this small neighbourhood one has $\left|\partial_{s}^{2} \Phi(s)\right|+\left|\partial_{s}^{2} \Phi(s)\right| \geqslant C_{0}$ and hence we may argue as in Case 2 of $\S 4.2$, obtaining the estimate

$$
\left|\mathcal{A}_{1}\right| \leqslant C(|\lambda| \nu)^{(\alpha-n \beta) / 2(\beta+1)} .
$$

We have therefore established that

$$
\mathcal{A}=C(|\lambda| \nu)^{(\alpha-(n-(1 / 6)) \beta) / 2(\beta+1)} \exp \left\{\mathrm{i}(|\lambda| \nu)^{\beta / 2(\beta+1)} \tilde{\Phi}\left(s_{0}\right)\right\}+O\left((|\lambda| \nu)^{(\alpha-n \beta) / 2(\beta+1)}\right),
$$

and hence we have also established the same equality for $\mathcal{I}_{3}$.

\subsection{Neighbourhood of the turning point: $|\nu-x| \leqslant \nu^{1 / 3}$}

Here we just use a size estimate and the fact that $\left|\Lambda_{k}^{\delta}(x)\right| \leqslant C \nu^{-1 / 3}$. This is the best we can do, since $\nu \phi^{3 / 2} \leqslant \nu((\nu-x) / \nu)^{3 / 2} \leqslant 1$.

Case 1. $(x \nu)^{1 / 2} \leqslant C_{1} r^{-\beta}$. We must integrate by parts and, since $x \leqslant C(x \nu)^{1 / 2}$, it suffices to estimate

$$
\begin{aligned}
\left|I_{4}\right| & =\left|\int_{0}^{\infty} \chi_{4}^{\varepsilon}(r) r^{-1-\alpha+N \beta} \mathrm{e}^{\mathrm{i} r^{-\beta}}(x \nu)^{(N+1-n) / 2} \Lambda_{k-N}^{n-1+N}(x) \mathrm{d} r\right| \\
& \leqslant C(|\lambda| \nu)^{(\alpha-n \beta) / 2(\beta+1)} \int_{|\nu-x| \leqslant \nu^{1 / 3}} \nu^{-1 / 3} \mathrm{~d} x \\
& \leqslant C(|\lambda| \nu)^{(\alpha-n \beta) / 2(\beta+1)},
\end{aligned}
$$

provided that $N$ is taken to be sufficiently large.

Case 2. $(x \nu)^{1 / 2} \geqslant C_{1} r^{-\beta}$. Here we will not integrate by parts first, so we wish to estimate

$$
\begin{aligned}
\left|\mathcal{I}_{4}\right| & =\left|\int_{0}^{\infty} \chi_{4}^{\varepsilon}(r) r^{-1-\alpha} \mathrm{e}^{\mathrm{i} r^{-\beta}}(x \nu)^{(1-n) / 2} \Lambda_{k}^{n-1}(x) \mathrm{d} r\right| \\
& \leqslant C(|\lambda| \nu)^{(\alpha-n \beta) / 2(\beta+1)} \int_{|\nu-x| \leqslant \nu^{1 / 3}} \nu^{-1 / 3} \mathrm{~d} x \\
& \leqslant C(|\lambda| \nu)^{(\alpha-n \beta) / 2(\beta+1)} .
\end{aligned}
$$




\subsection{Monotonic region I: $\nu+\nu^{1 / 3} \leqslant x \leqslant \frac{3}{2} \nu$}

Recall that here $\left|\Lambda_{k}^{\delta}(x)\right| \leqslant C \nu^{-1 / 4}(x-\nu)^{-1 / 4} \exp \left\{-\gamma_{1} \nu^{-1 / 2}(x-\nu)^{3 / 2}\right\}$.

Case 1. $(x \nu)^{1 / 2} \leqslant C_{1} r^{-\beta}$. In this region we should integrate by parts and, since $x \leqslant C(x \nu)^{1 / 2}$, it suffices to estimate

$$
\begin{aligned}
\left|I_{5}\right| & =\left|\int_{0}^{\infty} \chi_{5}^{\varepsilon}(r) r^{-1-\alpha+N \beta} \mathrm{e}^{\mathrm{i}^{-\beta}}(x \nu)^{(N+1-n) / 2} \Lambda_{k-N}^{n-1+N}(x) \mathrm{d} r\right| \\
& \leqslant C(|\lambda| \nu)^{(\alpha-n \beta) / 2(\beta+1)} \int_{x-\nu \geqslant \nu^{1 / 3}} \nu^{-1 / 4}(x-\nu)^{-1 / 4} \mathrm{e}^{-\gamma_{1} \nu^{-1 / 2}(x-\nu)^{3 / 2}} \mathrm{~d} x \\
& \leqslant C(|\lambda| \nu)^{(\alpha-n \beta) / 2(\beta+1)} \int_{u \geqslant 1} u^{-1 / 4} \mathrm{e}^{-\gamma_{1} u^{3 / 2}} \mathrm{~d} u \\
& \leqslant C(|\lambda| \nu)^{(\alpha-n \beta) / 2(\beta+1)}
\end{aligned}
$$

provided that $N$ is chosen to be sufficiently large.

Case 2. $(x \nu)^{1 / 2} \geqslant C_{1} r^{-\beta}$. Here we will not integrate by parts first, so it suffices to estimate

$$
\begin{aligned}
\left|\mathcal{I}_{5}\right| & =\left|\int_{0}^{\infty} \chi_{5}^{\varepsilon}(r) r^{-1-\alpha} \mathrm{e}^{\mathrm{i} r^{-\beta}}(x \nu)^{(1-n) / 2} \Lambda_{k}^{n-1}(x) \mathrm{d} r\right| \\
& \leqslant C(|\lambda| \nu)^{(\alpha-n \beta) / 2(\beta+1)} \int_{x-\nu \geqslant \nu^{1 / 3}} \nu^{-1 / 4}(x-\nu)^{-1 / 4} \mathrm{e}^{-\gamma_{1} \nu^{-1 / 2}(x-\nu)^{3 / 2}} \mathrm{~d} x \\
& \leqslant C(|\lambda| \nu)^{(\alpha-n \beta) / 2(\beta+1)},
\end{aligned}
$$

as before.

\subsection{Monotonic region II: $x \geqslant \frac{3}{2} \nu$}

Here we have the trivial estimate $\left|\Lambda_{k}^{\delta}(x)\right| \leqslant C \mathrm{e}^{-\gamma_{2} x}$.

Case 1. $x \leqslant C_{1} r^{-\beta}$. We should integrate by parts and since $x \geqslant(x \nu)^{1 / 2}$ it suffices to estimate

$$
\begin{aligned}
\left|I_{6}\right| & =\left|\int_{0}^{\infty} \chi_{6}^{\varepsilon}(r) r^{-1-\alpha+N \beta} \mathrm{e}^{\mathrm{i} r^{-\beta}} x^{N}(x \nu)^{(1-n) / 2}\right| \Lambda_{k}^{n-1}(x)|\mathrm{d} r| \\
& \leqslant C|\lambda|^{(\alpha-N \beta) /(\beta+2)} \nu^{1-n} \int_{x \geqslant \nu} x^{N-1} \mathrm{e}^{-\gamma_{2} x} \mathrm{~d} x \\
& \leqslant C|\lambda|^{(\alpha-N \beta) /(\beta+2)} \nu^{N-n} \mathrm{e}^{-\gamma_{2} \nu},
\end{aligned}
$$

for all $N$ sufficiently large. 
Case 2. $x \geqslant C_{1} r^{-\beta}$. Here we will not integrate by parts first, so it suffices to estimate

$$
\begin{aligned}
\left|\mathcal{I}_{6}\right| & =\left|\int_{0}^{\infty} \chi_{6}^{\varepsilon}(r) r^{-1-\alpha} \mathrm{e}^{\mathrm{i} r^{-\beta}}(x \nu)^{(1-n) / 2} \Lambda_{k}^{n-1}(x) \mathrm{d} r\right| \\
& \leqslant C|\lambda|^{(\alpha-N \beta) /(\beta+2)} \nu^{1-n} \int_{x \geqslant \nu} x^{N-1} \mathrm{e}^{-\gamma_{2} x} \mathrm{~d} x \\
& \leqslant C|\lambda|^{(\alpha-N \beta) /(\beta+2)} \nu^{N-n} \mathrm{e}^{-\gamma_{2} \nu},
\end{aligned}
$$

for all $N \geqslant 0$.

This completes the proof of Theorem 2.1.

\section{Proof of Theorem 1.4}

\subsection{Establishing sufficiency}

We noted above that Theorem 1.1 is essentially no more than a special case of Theorem 1.4: the case $\lambda=0$. The sufficiency in Theorem 1.4 is, however, as we shall see, an almost immediate consequence of that in Theorem 1.1 and it is to this that we now turn our attention.

\subsubsection{Establishing sufficiency in Theorem 1.1}

We may clearly assume that our kernel $K_{\alpha, \beta}(x, y)$ is supported in a small neighbourhood of the diagonal, as in the complement of such a region $K_{\alpha, \beta}$ is, for $\alpha>0$, clearly dominated by an integrable function of $|x-y|$.

In order to establish the positive result, we will dyadically decompose the operator

$$
T=\sum_{j=0}^{\infty} T_{j} .
$$

In order to do this we consider the following partition of unity; choose $\vartheta \in C_{0}^{\infty}(\mathbb{R})$ supported in $\left[\frac{1}{2}, 2\right]$ such that $\sum_{j=0}^{\infty} \vartheta\left(2^{j} r\right)=1$ for all $0 \leqslant r \leqslant 1$, and then, for $f \in \mathcal{S}$ with compact support, write

$$
T_{j} f(x)=\int K_{j}(x, y) f(y) \mathrm{d} y
$$

whenever $x$ is in the complement of the support of $f$, where

$$
K_{j}(x, y)=\vartheta\left(2^{j}|x-y|\right) K_{\alpha, \beta}(x, y) .
$$

The key result here is the following theorem.

Theorem 5.1. The operator norms of $T_{j}$ are uniformly bounded whenever $\alpha \leqslant \frac{1}{2}(d \beta)$; more precisely,

$$
\int_{\mathbb{R}^{d}}\left|T_{j} f(x)\right|^{2} \mathrm{~d} x \leqslant C 2^{j(2 \alpha-d \beta)} \int_{\mathbb{R}^{d}}|f(x)|^{2} \mathrm{~d} x .
$$


We note that, as the operator norms of $T_{j}$ are equal to that of

$$
\tilde{T}_{j} f(x)=2^{j \alpha} \int_{\mathbb{R}^{d}} \vartheta(|x-y|) a_{2^{-j}}(x, y) \mathrm{e}^{\mathrm{i} 2^{j \beta} \varphi_{2^{-j}}(x, y)} f(y) \mathrm{d} y,
$$

it suffices to consider the operators $\tilde{T}_{j}$ and to establish the inequality

$$
\int_{\left|x-x_{0}\right| \leqslant 1}\left|\tilde{T}_{j} f(x)\right|^{2} \mathrm{~d} x \leqslant C 2^{j(2 \alpha-d \beta)} \int_{\left|x-x_{0}\right| \leqslant 10}|f(x)|^{2} \mathrm{~d} x
$$

uniformly for all $x_{0}$ in $\mathbb{R}^{d}$. Integrating (5.1) with respect to $x_{0}$ then gives Lemma 5.1.

The key to establishing (5.1) is the following proposition of Hörmander, which may be thought of as a variable coefficient version of Plancherel's theorem. (For a proof see $[\mathbf{9}$, Chapter IX].)

Proposition 5.2. Let $\Psi$ be a smooth function of compact support in $x$ and $y$ and let $\Phi$ be real valued and smooth. If we assume that

$$
\operatorname{det}\left(\frac{\partial^{2} \Phi}{\partial x_{i} \partial y_{j}}\right) \neq 0
$$

on the support of $\Psi$, then

$$
\left\|\int_{\mathbb{R}^{d}} \Psi(x, y) \mathrm{e}^{\mathrm{i} \lambda \Phi(x, y)} f(y) \mathrm{d} y\right\|_{L^{2}\left(\mathbb{R}^{d}\right)} \leqslant C \lambda^{-d / 2}\|f\|_{L^{2}\left(\mathbb{R}^{d}\right)} .
$$

Proof of equation (5.1). We will first assume that $x_{0}=0$ and write $f=f_{1}+f_{2}$, with $f_{1}$ supported in $B(10), f_{2}$ supported outside $B(9), f_{1}$ and $f_{2}$ smooth, and with $\left|f_{1}\right|,\left|f_{2}\right| \leqslant|f|$. We fix $\chi \in C_{0}^{\infty}$ so that $\chi \equiv 1$ in $B(1)$.

Now, since the integral kernel of $\tilde{T}_{j}$ is compactly supported in $x-y$, it follows that $\chi \tilde{T}_{j}$ is compactly supported in $x$ and $y$. Therefore, applying Proposition 5.2, we see that

$$
\begin{aligned}
\int_{B(1)}\left|\tilde{T}_{j} f_{1}(x)\right| \mathrm{d} x & =\int_{\mathbb{R}^{d}}\left|\chi(x) \tilde{T}_{j} f_{1}(x)\right| \mathrm{d} x \\
& \leqslant C 2^{j(2 \alpha-d \beta)} \int_{\mathbb{R}^{d}}\left|f_{1}(x)\right|^{2} \mathrm{~d} x \\
& \leqslant C 2^{j(2 \alpha-d \beta)} \int_{B(10)}|f(x)|^{2} \mathrm{~d} x .
\end{aligned}
$$

However, if $|x| \leqslant 1$, and $|y| \geqslant 9$, it follows that $|x-y| \geqslant 8$ and, hence, $\chi \tilde{T}_{j} f_{2} \equiv 0$.

The passage to general $x_{0}$ is then easy since, although $\tilde{T}_{j}$ are not translation invariant, the 'translated' kernels

$$
K_{\alpha, \beta}^{x_{0}}(x, y)=K_{\alpha, \beta}\left(x+x_{0}, y+x_{0}\right)
$$


do satisfy assumptions (1.2) and (1.3), for the same $\alpha$ and $\beta$ as $K_{\alpha, \beta}$, uniformly in $x_{0}$, and hence

$$
\begin{aligned}
\int_{\left|x-x_{0}\right| \leqslant 1}\left|\tilde{T}_{j} f(x)\right|^{2} \mathrm{~d} x & =\int_{\left|x-x_{0}\right| \leqslant 1}\left|2^{-j d} \int K_{j}\left(2^{-j} x, 2^{-j} y\right) f(y) \mathrm{d} y\right|^{2} \mathrm{~d} x \\
& =\int_{|x| \leqslant 1}\left|2^{-j d} \int K_{j}^{x_{0}}\left(2^{-j} x, 2^{-j} y\right) f\left(y+x_{0}\right) \mathrm{d} y\right|^{2} \mathrm{~d} x \\
& \leqslant C 2^{j(2 \alpha-d \beta)} \int_{|x| \leqslant 10}\left|f\left(x+x_{0}\right)\right|^{2} \mathrm{~d} x \\
& \leqslant C 2^{j(2 \alpha-d \beta)} \int_{\left|x-x_{0}\right| \leqslant 10}|f(x)|^{2} \mathrm{~d} x,
\end{aligned}
$$

where $K_{j}^{x_{0}}(x, y)=\vartheta\left(2^{j}(x-y)\right) K_{\alpha, \beta}^{x_{0}}(x, y)$.

Theorem 1.1 now follows from Lemma 5.1 and an application of Cotlar's lemma (plus a standard limiting argument) once we have verified that the $T_{j}$ are, in the following sense, almost orthogonal.

Lemma 5.3. If $\alpha=\frac{1}{2} d \beta$, then $\left\|T_{i}^{*} T_{j}\right\|_{O p}+\left\|T_{i} T_{j}^{*}\right\|_{O p} \leqslant C 2^{-(d \beta / 2)|i-j|}$.

Proof. This follows trivially from Lemma 5.1 whenever $|i-j| \leqslant 10$, since $\left\|T_{i}^{*} T_{j}\right\|_{O p} \leqslant$ $\left\|T_{i}\right\|_{O p}\left\|T_{j}\right\|_{O p}$. We will therefore, without loss of generality, assume that $j \geqslant i+10$. Now $T_{i}^{*} T_{j}$ has a kernel

$$
L_{i j}(x, y)=\int \bar{K}_{i}(z, x) K_{j}(z, y) \mathrm{d} z
$$

and the same operator norm as the operator with kernel

$$
\begin{aligned}
\tilde{L}_{i j}(x, y) & =2^{-j d} L_{i j}\left(2^{-j} x, 2^{-j} y\right) \\
& =2^{-j d} \int \bar{K}_{i}\left(z, 2^{-j} x\right) K_{j}\left(z, 2^{-j} y\right) \mathrm{d} z \\
& =2^{j 2 \alpha} \int_{\substack{|z-y| \sim 1 \\
|z-x| \sim 2^{j-i}}} a_{2^{-j}}(z, x) a_{2^{-j}}(z, y) \exp \left\{\mathrm{i}^{j \beta}\left[\varphi_{2^{-j}}(z, y)-\varphi_{2^{-j}}(z, x)\right]\right\} \mathrm{d} z .
\end{aligned}
$$

Trivially we get the estimate $\left|\tilde{L}_{i j}(x, y)\right| \leqslant C 2^{j 2 \alpha} 2^{(i-j)(d+\alpha)}$. However, from $(1.2 c)$ it follows that

$$
\left|\nabla_{z}\left[\varphi_{2^{-j}}(z, y)-\varphi_{2^{-j}}(z, x)\right]\right| \geqslant C_{0} .
$$

Thus, there is always a direction in which we may integrate by parts; in doing so $d$ times we obtain

$$
\left|\tilde{L}_{i j}(x, y)\right| \leqslant C 2^{j(2 \alpha-d \beta)} 2^{(i-j)(d+\alpha)}=2^{(i-j)(d+\alpha)} .
$$

This of course implies that

$$
\sup _{x} \int\left|\tilde{L}_{i j}(x, y)\right| \mathrm{d} y \leqslant C 2^{(i-j) \alpha}
$$


and

$$
\sup _{y} \int\left|\tilde{L}_{i j}(x, y)\right| \mathrm{d} x \leqslant C 2^{(i-j) \alpha}
$$

and so, by Schur's lemma, we are done.

\subsubsection{Establishing sufficiency in Theorem 1.4}

The task of proving the positive half of Theorem 1.4 reduces, as above, to establishing the following result.

Theorem 5.4. The inequality

$$
\int_{\left|z-z_{0}\right| \leqslant 1}\left|T^{\lambda} f(z)\right|^{2} \mathrm{~d} z \leqslant C_{\lambda} \int_{\left|z-z_{0}\right| \leqslant 10}|f(z)|^{2} \mathrm{~d} z
$$

holds, with constant $C_{\lambda}$ independent of $z_{0}$.

As before, Theorem 1.4 then follows immediately from Theorem 5.4 via an integration in $z_{0}$.

Proof. The case when $\lambda=0$ of course follows from estimate (5.1) above, so in what follows it is understood that $\lambda \neq 0$. Using the fact that, for $z, w \in \mathbb{C}^{n}$, we have

$$
\left.\mid \mathrm{e}^{\mathrm{i}(\lambda / 2) \operatorname{Im} z \cdot \bar{w}}-\sum_{k=0}^{N-1} \frac{\lambda^{k}}{4^{k} k !} \overline{((z-w)} \cdot w-(z-w) \cdot \bar{w}\right)\left.^{k}\left|\leqslant C_{\lambda}\right| w\right|^{N}|z-w|^{N},
$$

we see that matters reduce to estimating operators of the form

$$
f \mapsto \int_{\mathbb{C}^{n}} \mathcal{K}(z, w) f(w) \mathrm{d} w
$$

where

$$
\mathcal{K}(z, w)=K_{\alpha, \beta}(z, w)(\bar{z}-\bar{w})^{\ell}(z-w)^{m}
$$

for $|\ell|+|m|=k=0, \ldots, N-1$.

It is possible to then establish that these operators are bounded in $L^{2}\left(\mathbb{C}^{n}\right)$ whenever $\alpha-k \leqslant n \beta$ in the model case by appealing to spherical harmonics and Fourier transform methods. It is, however, easy to see that these kernels $\mathcal{K}(z, w)$ are in fact strongly singular integral kernels and it therefore follows immediately from Theorem 1.1, in particular inequality (5.1), that these more general operators are also bounded in $L^{2}\left(\mathbb{C}^{n}\right)$ whenever $\alpha-k \leqslant n \beta$. This establishes Theorem 5.4 and hence the sufficiency of $\alpha \leqslant n \beta$ in Theorem 1.4 .

\subsection{Establishing necessity}

We begin by noting that the model kernels $K_{\alpha, \beta}(x, y)=\tilde{K}_{\alpha, \beta}(x-y)$ are indeed strongly singular integral kernels. The fact that they satisfy the differential inequalities (1.2) is self-evident and the simple lemma below establishes that they also satisfy the required non-degeneracy hypothesis. 
Lemma 5.5. Let $\varphi(x, y)=|x-y|^{-\beta}$. Then

$$
\operatorname{det}\left(\frac{\partial^{2} \varphi}{\partial x_{i} \partial y_{j}}\right) \neq 0
$$

whenever $\beta \neq-1$.

Proof. Recall that

$$
\nabla|x|^{-\beta}=-\beta|x|^{-\beta-1} \frac{x}{|x|} .
$$

It is then easy to see that

$$
\partial_{x_{i}} \partial_{y_{j}} \varphi(x, y)=\beta|x-y|^{-\beta-2}\left(\delta_{i j}-(\beta+2) u_{i} u_{j}\right),
$$

where $u_{i}=(x-y)_{i} /|x-y|$. We therefore need to check that $I-(\beta+2) u u^{T}$ is nonsingular. To do this we will denote by $R$ the rotation matrix such that $R u=e_{1}$; of course, $\operatorname{det} R=1$ and it is clear that

$$
\operatorname{det}\left(I-(\beta+2) u u^{\mathrm{T}}\right)=\operatorname{det}\left(R\left(I-(\beta+2) u u^{\mathrm{T}}\right) R^{\mathrm{T}}\right)=1-(\beta+2)=-(\beta+1) .
$$

In order to establish the necessity of the condition $\alpha \leqslant n \beta$, it therefore suffices to test our model twisted convolution operator $R^{\lambda}$ on a suitably chosen $L^{2}\left(\mathbb{C}^{n}\right)$ function $f_{0}$ over an appropriately chosen range of $z$. We choose as our test function $f_{0}(z)=|z|^{-\gamma} \chi(10|z|)$, where $\gamma<n$. Restricting ourselves to small $|z|$, we therefore formally have

$$
\begin{aligned}
\chi(10|z|) & R^{\lambda} f_{0}(z) \\
& =\chi(10|z|) \int_{\mathbb{C}^{n}}|z-w|^{-2 n-\alpha} \exp \left\{\mathrm{i}\left(|z-w|^{-\beta}+\frac{1}{2} \lambda \operatorname{Im} z \cdot \bar{w}\right)\right\} \chi(10|w|)|w|^{-\gamma} \mathrm{d} w .
\end{aligned}
$$

At this point we also make the observation that $R^{\lambda} f_{0}$ is a radial function and, as such, we may assume, with no loss in generality, that $z=(|z|, 0, \ldots, 0)$.

Now making the change of variables $w=|z| s$, we see that

$$
\chi(10|z|) R^{\lambda} f_{0}(z)=\chi(10|z|)|z|^{-\alpha-\gamma} \int_{\mathbb{C}^{n}} \exp \left\{\mathrm{i}|z|^{-\beta} \varphi(s,|z|)\right\} \psi(s) \mathrm{d} s,
$$

where

$$
\varphi(s,|z|)=\left(1-2 s_{1}+|s|^{2}\right)^{-\beta / 2}+\frac{1}{2} \lambda|z|^{\beta+2} s_{2}
$$

and

$$
\psi(s)=\left(1-2 s_{1}+|s|^{2}\right)^{-n-(\alpha / 2)} \chi(10|s|)|s|^{-\gamma} .
$$

We have therefore now reduced matters to the analysis of the oscillatory integral

$$
I(|z|)=\int_{\mathbb{C}^{n}} \exp \left\{\mathrm{i}|z|^{-\beta} \varphi(s,|z|)\right\} \psi(s) \mathrm{d} s,
$$


as $|z| \rightarrow 0$. We now write

$$
I(|z|)=M(|z|)+E_{1}(|z|)+E_{2}(|z|)+E_{3}(|z|),
$$

where

$$
\begin{aligned}
& M(|z|)=\mathrm{e}^{\mathrm{i}|z|^{-\beta}} \int_{\mathbb{C}^{n}} \chi\left(|z|^{-\beta(1-\varepsilon)}|s|\right) \exp \left\{\mathrm{i}\left[\beta|z|^{-\beta} s_{1}+\frac{1}{2} \lambda|z|^{2} s_{2}\right]\right\}|s|^{-\gamma} \mathrm{d} s, \\
& E_{1}(|z|)=\int_{\mathbb{C}^{n}} \chi\left(|z|^{-\beta(1-\varepsilon)}|s|\right) \exp \left\{\mathrm{i} \frac{1}{2} \lambda|z|^{2} s_{2}\right\} \\
& \quad \times\left[\exp \left\{\mathrm{i}|z|^{-\beta}\left(1-2 s_{1}+|s|^{2}\right)^{-\beta / 2}\right\}-\exp \left\{\mathrm{i}|z|^{-\beta}\left(1+\beta s_{1}\right)\right\}\right]|s|^{-\gamma} \mathrm{d} s, \\
& E_{2}(|z|)=\int_{\mathbb{C}^{n}} \chi\left(|z|^{-\beta(1-\varepsilon)}|s|\right) \mathrm{e}^{\mathrm{i}|z|^{-\beta} \varphi(s,|z|)}\left[\psi(s)-|s|^{-\gamma}\right] \mathrm{d} s
\end{aligned}
$$

and

$$
E_{3}(|z|)=\int_{\mathbb{C}^{n}}\left[1-\chi\left(|z|^{-\beta(1-\varepsilon)}|s|\right)\right] \mathrm{e}^{\mathrm{i}|z|^{-\beta} \varphi(s,|z|)} \psi(s) \mathrm{d} s .
$$

with $\varepsilon>0$ fixed sufficiently small.

Let us first take care of the error terms. It is easy to verify that whenever $|s| \leqslant|z|^{\beta(1-\varepsilon)}$ we have

$$
\left|\exp \left\{\mathrm{i}|z|^{-\beta}\left(1-2 s_{1}+|s|^{2}\right)^{-\beta / 2}\right\}-\exp \left\{\mathrm{i}|z|^{-\beta}\left(1+c s_{1}\right)\right\}\right| \leqslant C|z|^{-\beta \varepsilon}|s|
$$

and

$$
\left.\left.|\psi(s)-| s\right|^{-\gamma}|\leqslant C| z\right|^{\beta(1-\varepsilon)}|s|^{-\gamma}
$$

and hence that

$$
\begin{aligned}
\left|E_{1}(|z|)\right| & \leqslant C|z|^{-\beta \varepsilon} \int_{|s| \leqslant|z|^{\beta(1-\varepsilon)}}|s|^{-\gamma+1} \mathrm{~d} s \\
& \leqslant C|z|^{-\beta \varepsilon}|z|^{\beta(2 n-\gamma+1)(1-\varepsilon)} \\
& =C|z|^{\beta(2 n-\gamma)}|z|^{\beta(1-\varepsilon(2+2 n-\gamma))}
\end{aligned}
$$

while

$$
\begin{aligned}
\left|E_{2}(|z|)\right| & \leqslant C|z|^{\beta(1-\varepsilon)} \int_{|s| \leqslant|z|^{\beta(1-\varepsilon)}}|s|^{-\gamma} \mathrm{d} s \\
& \leqslant C|z|^{\beta(1-\varepsilon)}|z|^{\beta(2 n-\gamma)(1-\varepsilon)} \\
& =C|z|^{\beta(2 n-\gamma)}|z|^{\beta(1-\varepsilon(1+2 n-\gamma))} .
\end{aligned}
$$

In the error integral $E_{3}(|z|)$ it will be advantageous to repeatedly apply integration by parts in the $s_{1}$ direction since $C|z|^{\beta(1-\varepsilon)} \leqslant|s| \leqslant \frac{1}{10}$. In fact, it is clear that in this region

$$
\partial_{1} \varphi(s,|z|)=\beta\left(1-s_{1}\right)\left(1-2 s_{1}+|s|^{2}\right)^{-(\beta+2) / 2} \geqslant C(\beta)
$$


while $\left|\partial_{1}^{\ell} \varphi(s,|z|)\right| \leqslant c_{\ell}$, for all $\ell \geqslant 0$, and

$$
\left|\partial_{1}^{\ell}\left[1-\chi\left(|z|^{-\beta(1-\varepsilon)}|s|\right)\right] \psi(s)\right| \leqslant c_{\ell}\left(|z|^{-\beta(1-\varepsilon) \ell}|s|^{-\gamma} \vartheta\left(10|z|^{-\beta(1-\varepsilon)}|s|\right)+|s|^{-\gamma-\ell}\right) .
$$

It therefore follows that, after integrating by parts $N$ times, we obtain the estimate

$$
\begin{aligned}
\left|E_{3}(|z|)\right| & \leqslant C|z|^{\beta N}\left(|z|^{\beta(1-\varepsilon) N} \int_{|s| \approx|z|^{\beta(1-\varepsilon)}}|s|^{-\gamma} \mathrm{d} s+\int_{|s| \geqslant|z|^{\beta(1-\varepsilon)}}|s|^{-\gamma-N} \mathrm{~d} s\right) \\
& \leqslant C|z|^{\beta(2 n-\gamma)}|z|^{\beta \varepsilon(N-2 n+\gamma)} .
\end{aligned}
$$

It remains for us to show that, for $|z|$ sufficiently small,

$$
|M(|z|)| \geqslant C|z|^{\beta(2 n-\gamma)}
$$

Assuming this for the moment, we see that it would then follow that $|I(|z|)| \geqslant|z|^{\beta(2 n-\gamma)}$ for sufficiently small $|z|$, and, hence, that

$$
\left\|R^{\lambda} f_{0}\right\|_{2}^{2} \geqslant C \int_{\mathbb{C}^{n}} \chi(10|z|)|z|^{-2(\alpha+\gamma-\beta(2 n-\gamma))} \mathrm{d} z .
$$

It then follows that if $R^{\lambda}$ were to extend to a bounded operator on $L^{2}\left(\mathbb{C}^{n}\right)$, we must have

$$
\alpha-n \beta<(n-\gamma)(\beta+1)
$$

for all $\gamma<n$. It follows immediately that we must then necessarily have the condition $\alpha \leqslant n \beta$.

The lower bound estimate (5.2) for the main term will be an almost immediate consequence of the following, slightly more general lemma.

Lemma 5.6. If $\gamma<\frac{1}{2} d$, then

$$
\int_{\mathbb{R}^{d}} \chi(|s|) \mathrm{e}^{\mathrm{i} \xi \cdot s}|s|^{-\gamma} \mathrm{d} s=C|\xi|^{\gamma-d}+O\left(|\xi|^{\gamma-d-1}\right) .
$$

Let us assume that Lemma 5.6 holds for the moment and see how this gives us (5.2). To do this we first rescale the integral $M(|z|)$ so that

$$
M(|z|)=|z|^{(2 n-\gamma) \beta(1-\varepsilon)} \mathrm{e}^{\mathrm{i}|z|^{-\beta}} \int_{\mathbb{C}^{n}} \chi(|s|) \exp \left\{\mathrm{i}\left[c|z|^{-\beta \varepsilon} s_{1}+\frac{1}{2} \lambda|z|^{2+\beta(1-\varepsilon)} s_{2}\right]\right\}|s|^{-\gamma} \mathrm{d} s .
$$

Applying Lemma 5.6 to this then gives

$$
\begin{aligned}
M(|z|)=|z|^{(2 n-\gamma) \beta} \mathrm{e}^{\mathrm{i}|z|^{-\beta}}\left(1+\left(\frac{1}{2} \lambda|z|^{\beta+2}\right)^{2}\right)^{(\gamma-2 n) / 2} & \\
& +O\left(|z|^{(2 n-\gamma+\varepsilon) \beta}\left(1+\left(\frac{1}{2} \lambda|z|^{\beta+2}\right)^{2}\right)^{(\gamma-2 n-1) / 2}\right) .
\end{aligned}
$$

The result now follows if we initially restrict ourselves to $|z| \leqslant \min \left\{\frac{1}{100}, \lambda^{-1 /(\beta+2)}\right\}$. 
Proof of Lemma 5.6. This is merely a Fourier transform, and hence

$$
\int_{\mathbb{R}^{d}} \chi(|s|) \mathrm{e}^{\mathrm{i} \xi \cdot s}|s|^{-\gamma} \mathrm{d} s=C \int_{\mathbb{R}^{d}} \hat{\chi}(|\eta-\xi|)|\eta|^{\gamma-d} \mathrm{~d} \eta .
$$

Now since $\chi$ is smooth and of compact support $\hat{\chi}$ is a Schwartz function and satisfies the inequality

$$
|\hat{\chi}(|\eta-\xi|)| \leqslant C_{N}(1+|\eta-\xi|)^{-N}
$$

for all $N \geqslant 0$. Using this standard estimate it is easy to see that whenever $|\xi| \notin\left[\frac{1}{2}|\eta|, 2|\eta|\right]$ we have

$$
\left.\left.\left|\int_{\mathbb{R}^{d}} \hat{\chi}(|\eta-\xi|)\right| \eta\right|^{\gamma-d} \mathrm{~d} \eta|\leqslant C| \xi\right|^{-N+\gamma} .
$$

Now, if $|\xi| \in\left[\frac{1}{2}|\eta|, 2|\eta|\right]$, then

$$
\begin{aligned}
\int_{\mathbb{R}^{d}} \hat{\chi}(|\eta-\xi|)|\eta|^{\gamma-d} \mathrm{~d} \eta & =|\xi|^{\gamma-d} \int_{\mathbb{R}^{d}} \hat{\chi}(|\eta-\xi|) \mathrm{d} \eta+\int_{\mathbb{R}^{d}} \hat{\chi}(|\eta-\xi|)\left[|\eta|^{\gamma-d}-|\xi|^{\gamma-d}\right] \mathrm{d} \eta \\
& =|\xi|^{\gamma-d} \chi(0)+O\left(\int_{|\eta| \approx|\xi|}|\eta|^{\gamma-d-1} \mathrm{~d} \eta\right) \\
& =|\xi|^{\gamma-d}+O\left(|\xi|^{\gamma-d-1}\right) .
\end{aligned}
$$

\section{Final remarks}

\subsection{Extensions}

Using similar methods, we can also establish Theorem 1.2 for the integral operators

$$
R_{\gamma} f(z, t)=\int_{\mathbb{H}^{n}} M_{\gamma}\left([w, s]^{-1} \cdot[z, t]\right) f(w, s) \mathrm{d} w \mathrm{~d} s,
$$

where

$$
M_{\gamma}(z, t)=\tilde{K}_{\alpha, \beta}(z) \delta_{0}\left(t-|z|^{\gamma}\right)
$$

with $\gamma \geqslant 2$, and Theorem 1.4 for the integral operators

$$
T_{\gamma}^{\lambda} f(z)=\int_{\mathbb{C}^{n}} K_{\alpha, \beta}(z, w) \mathrm{e}^{\mathrm{i} \lambda|z-w|^{\gamma}} \exp \left\{\mathrm{i} \frac{1}{2} \lambda \operatorname{Im} z \cdot \bar{w}\right\} f(w) \mathrm{d} w,
$$

with $\gamma \geqslant 0$.

We plan to discuss these operators, in particular the uniform behaviour of the operators $T_{\gamma}^{\lambda}$, in more detail in a future paper. 


\subsection{Strongly singular Radon transforms on $\mathbb{R}^{d+1}$}

We should finish by saying something about the analogous class of strongly singular Radon transforms on $\mathbb{R}^{d+1}$ formally given by

$$
\mathcal{R}_{\gamma} f=f * L,
$$

where $L(x, t)=\tilde{K}_{\alpha, \beta}(x) \delta_{0}\left(t-|x|^{\gamma}\right)$ and again $\gamma \geqslant 0$. The following result is due to Chandarana [2] (see also [14]).

Theorem 6.1. If $\gamma \geqslant 2$, then $\mathcal{R}_{\gamma}$ extends to a bounded operator on $L^{2}\left(\mathbb{R}^{2}\right)$ if and only if $\alpha \leqslant\left(\frac{1}{2}-\frac{1}{6}\right) \beta$.

We take this opportunity to remark that the situation when $d \geqslant 2$ is quite different.

Theorem 6.2. If $d \geqslant 2$ and $\gamma \geqslant 0$, then $\mathcal{R}_{\gamma}$ extends to a bounded operator on $L^{2}\left(\mathbb{R}^{d+1}\right)$ if and only if $\alpha \leqslant \frac{1}{2} \beta$.

As with the model operators discussed earlier, proving $L^{2}$-boundedness is equivalent to establishing the uniform boundedness, in $\mathbb{R}^{d+1}$, of the multiplier

$$
m(\xi, \lambda)=(2 \pi)^{d / 2} \int_{0}^{\infty} \chi(r) r^{-1-\alpha} \exp \left\{\mathrm{i}\left(r^{-\beta}+\lambda r^{\gamma}\right)\right\} J_{(d-2) / 2}(r|\xi|)(r|\xi|)^{(d-2) / 2} \mathrm{~d} r .
$$

It is then easy to see that, for $r|\xi|$ small and $\lambda$ large, the phase in this integral may be stationary and that we can, in this region, establish only the estimate

$$
|m(\xi, \lambda)| \leqslant C|\lambda|^{\alpha-(\beta / 2)} .
$$

When $d \geqslant 2$ this is in fact the worst region and one can, by applying the method of stationary phase, show that, for $\xi$ fixed,

$$
m(\xi, \lambda) \sim|\lambda|^{\alpha-(\beta / 2)} .
$$

Acknowledgements. The problems addressed in this paper were originally suggested by Fulvio Ricci and I thank him for his generosity, support and guidance. The argument used in the proof of Lemma 5.5 was shown to the author by Andreas Seeger. The necessity argument presented in $\S 5.2$ follows from a suggestion by Steve Wainger. I thank him for this and also for numerous other fruitful discussions.

\section{References}

1. R. Askey and S. Wainger, Mean convergence of expansions in Laguerre and Hermite series, Am. J. Math. 87 (1965), 695-708.

2. S. Chandarana, $L^{p}$-bounds for hypersingular integral operators along curves, Pac. J. Math. 175 (1996), 389-415.

3. A. ERDÉLYI, Asymptotic forms for Laguerre polynomials, J. Indian Math. Soc. 24 (1960), 235-250.

4. C. Fefferman, Inequalities for strongly singular convolution operators, Acta Math. 124 (1970), 9-36. 
5. C. Fefferman and E. M. Stein, $H^{p}$ spaces of several variables, Acta Math. 129 (1972), 137-193.

6. D. Geller, Fourier analysis on the Heisenberg group, Proc. Natl Acad. Sci. USA $\mathbf{7 4}$ (1977), 1328-1331.

7. I. I. Hinschman, Multiplier transforms, I, Duke Math. J. 26 (1956), 222-242.

8. N. LyalL, Strongly singular convolution operators on the Heisenberg group, Trans. Am. Math. Soc., in press.

9. E. M. StEIn, Harmonic analysis: real-variable methods, orthogonality, and oscillatory integrals (Princeton University Press, 1993).

10. E. M. Stein AND G. Weiss, Introduction to Fourier analysis on Euclidean spaces (Princeton University Press, 1971).

11. S. ThangaVelu, Harmonic analysis on the Heisenberg group (Birkhäuser, 1998).

12. S. WAinger, Special trigonometric series in $k$ dimensions, Memoirs of the American Mathematical Society, Volume 59 (American Mathematical Society, Providence, RI, 1965).

13. T. H. WolfF, Lectures on harmonic analysis, University Lecture Series, Volume 29 (American Mathematical Society, Providence, RI, 2003).

14. M. ZiELINSKI, Highly oscillatory integrals along curves, $\mathrm{PhD}$ thesis, University of Wisconsin-Madison (1985). 